\title{
Large p explorations. From SUGRA to big STRINGS in Mellin space
}

\author{
Francesco Aprile ${ }^{a}$ and Pedro Vieira ${ }^{b, c}$ \\ ${ }^{a}$ Dipartimento di Fisica, Università di Milano-Bicocca \& INFN, Sezione di Milano-Bicocca, \\ Piazza della Scienza, U2, I-20126 Milano, Italy \\ ${ }^{b}$ Perimeter Institute for Theoretical Physics, \\ 31 Caroline St N, Waterloo, Ontario N2L 2Y5, Canada \\ ${ }^{c}$ Instituto de Fisica Teorica, UNESP, ICTP South American Institute for Fundamental Research, \\ Rua Dr Bento Teobaldo Ferraz 271, São Paulo 01140-070, Brazil \\ E-mail: francesco.aprile1@unimib.it, pedrogvieira@gmail.com
}

ABSTRACT: We explore a new way of probing scattering of closed strings in $A d S_{5} \times S^{5}$, which we call 'the large $p$ limit'. It consists of studying four-point correlators of singleparticle operators in $\mathcal{N}=4 \mathrm{SYM}$ at large $N$ and large 't Hooft coupling $\lambda$, by looking at the regime in which the dual KK modes become short massive strings. In this regime the charge of the single-particle operators is order $\lambda^{1 / 4}$ and the dual KK modes are in between fields and strings. Starting from SUGRA we compute the large $p$ limit of the correlators by introducing an improved $A d S_{5} \times S^{5}$ Mellin space amplitude, and we show that the correlator is dominated by a saddle point. Our results are consistent with the picture of four geodesics shooting from the boundary of $A d S_{5} \times S^{5}$ towards a common bulk point, where they scatter as if they were in flat space. The Mandelstam invariants are put in correspondence with the Mellin variables and in turn with certain combinations of cross ratios. At the saddle point the dynamics of the correlator is directly related to the bulk Mellin amplitude, which in the process of taking large $p$ becomes the flat space ten-dimensional S-matrix. We thus learn how to embed the full type IIB S-matrix in the $A d S_{5} \times S^{5}$ Mellin amplitude, and how to stratify the latter in a large $p$ expansion. We compute the large $p$ limit of all genus zero data currently available, pointing out additional hidden simplicity of known results. We then show that the genus zero resummation at large $p$ naturally leads to the Gross-Mende phase for the minimal area surface around the bulk point. At one-loop, we first uncover a novel and finite Mellin amplitude, and then we show that the large $p$ limit beautifully asymptotes the gravitational S-matrix.

Keywords: 1/ $N$ Expansion, AdS-CFT Correspondence, Scattering Amplitudes

ArXiv EPrint: 2007.09176 


\section{Contents}

1 Introduction 1

2 Intuition from geodesics $\quad 5$

3 Genus zero amplitude $\quad 8$

3.1 Tree level SUGRA 8

$3.2 \quad$ Large $p$ limit $=10 \mathrm{~d}$ Virasoro-Shapiro $\quad 9$

3.3 Towards the full amplitude: $\zeta_{3}$ and $\zeta_{5} \quad 11$

4 One-loop SUGRA 12

$\begin{array}{lll}4.1 & \text { Logarithmic discontinuities } & 13\end{array}$

$\begin{array}{lll}4.2 & \text { One-loop Mellin amplitudes } & 14\end{array}$

$\begin{array}{lll}\text { 4.2.1 Top-weight } 4 & 15\end{array}$

$\begin{array}{lll}4.2 .2 & \text { Top-weight } 3 & 16\end{array}$

$\begin{array}{lll}4.2 .3 & \text { Top-weight } 2 & 17\end{array}$

4.3 Gravitational S-matrix in the large $p$ limit 20

5 Discussion $\quad 22$

A Holographic correlators and the large $p$ limit 26

A.1 Conventions on Mellin and Mandelstam variables 26

$\begin{array}{ll}\text { A.2 Conventions on SUGRA amplitudes } & 27\end{array}$

$\begin{array}{lll}\text { A.3 OPE view on the Mellin amplitude } & 29\end{array}$

B Mellin saddle point details $\quad 30$

B.1 Saddle point on $A d S_{5} \quad 30$

B.2 Saddle point on $S^{5}$

$\begin{array}{ll}\text { C Virasoro-Shapiro } & \mathbf{3 4}\end{array}$

C.1 Low energy expansion and asymptotics 34

C.2 More on the large $p$ limit 35

D Leading discontinuities from $\widehat{\mathcal{D}}(\vec{p})$ and $\Delta^{(8)} \quad 36$

$\begin{array}{lll}\text { D.1 Leading log at two-loop } & 39\end{array}$ 


\section{Introduction}

Half-BPS single trace gauge invariant operators

$$
T_{p} \equiv \operatorname{Tr}(\vec{Y} \cdot \phi(x))^{p}
$$

are probably the simplest conceivable operators in $\mathcal{N}=4$ SYM. A dream is to compute their correlation functions at any value of the 't Hooft coupling $\lambda$ and for any number of colours $N$. This would amount to computing a full closed string scattering amplitude in $A d S_{5} \times S^{5}$ for any string tension $(\sqrt{\lambda})$ and any string coupling $(1 / N)$. For four operators and at large $N$ - corresponding to the planar theory - this would be the AdS analogue of the flat space Virasoro-Shapiro amplitude. Despite the fact that finding this amplitude, and more generally gaining full control over the genus expansion of the theory, is still a formidable open problem, the recent years have witness an increasing number of important insights into this program. New ideas have come from the bootstrap approach $[1-5,8,10-$ 16] integrability [25, 26, 35-38] and localization [44-47].

Here we propose to consider the corner of parameters space in which $p$ is large as a way to explore closed string scattering in $A d S_{5} \times S^{5}$. Indeed, from the large $p$ limit we will learn an important lesson about how four-point correlation functions behave in general.

We consider the limit where $N$ is the largest parameter, and $\lambda$ is also large. Depending on how large $p$ is we have various different physical regimes:

$$
\begin{array}{ll}
p=O(1) & \text { SUGRA } \\
p=O\left(\lambda^{1 / 4}\right) & \text { short massive strings } \\
p=O\left(\lambda^{1 / 2}\right) & \text { big classical strings . }
\end{array}
$$

We will start with the correlators in SUGRA where $p=O(1)$ and take $p \gg 1$ in those results. We will see that they simplify dramatically but still retain a lot of non trivial information. In particular, in the domain $p=O\left(\lambda^{1 / 4}\right)$ we make contact with the low energy limit of the short massive string [23], and since we are dealing with near point-like objects, compared to the ambient space, we shall see that in this regime the strings scatter as in flat space.

When $p$ increases further, outside the short string domain, the strings start to open up and we reach the high energy scattering region dominated by the flat space result of Gross-Mende [24]. As $p=O(\sqrt{\lambda})$ the angular momenta of the strings is so huge that their centripetal forces open up the strings in the full $A d S_{5} \times S^{5}$. At this point integrability should kick in and allow us to compute the relevant minimal areas generalising to $A d S_{5} \times S^{5}$ the computation of Gross-Mende. We make a few comments about this short/long string transition in the conclusions.

The bulk of the paper is mostly about the transition between the SUGRA/short string transition and about the insights extracted from this analysis at fixed $p$. To analyse that, we focus on the dynamical part of the correlator

$$
\left\langle\mathcal{O}_{p_{1}}\left(x_{1}\right) \ldots \mathcal{O}_{p_{4}}\left(x_{4}\right)\right\rangle_{\text {connected }}=(\text { free theory })+(\text { kinematics }) \times \mathcal{A}_{\vec{p}}(U, V, \tilde{U}, \tilde{V})
$$


which is what we call the amplitude factor $\mathcal{A}_{\vec{p}}$. The superconformal splitting of the correlator [39], and the kinematic elements, are standard and detailed in appendix A.2. Let us highlight here a few important elements about this starting point:

The operators $\mathcal{O}_{p}$ are the single particle operators (SPOs) defined in [15], and are the half-BPS operators properly dual to the Kaluza Klein modes on the $A d S_{5} \times S^{5}$ background. SPOs coincide with the single trace operators $T_{p}$ in (1.1) the strict large $N$ limit, but in general are given by an admixture of single and multi-trace operators of the form

$$
\mathcal{O}_{p}=T_{p}+\sum_{q_{1}+q_{2}=p} C_{q_{1} q_{2}}(N) T_{q_{1}} T_{q_{2}}+\sum_{q_{1}+q_{2}+q_{3}=p} C_{q_{1} q_{2} q_{3}}(N) T_{q_{1}} T_{q_{2}} T_{q_{3}}+\ldots
$$

The various terms correspond to all possible partitions of the charge $p$. At finite $N$, the coefficient $C_{\left\{q_{i}\right\}}$ are obtained by imposing that the two point functions of $\mathcal{O}_{p}$ with any other multi-trace operator, they all vanish. These coefficients, and other surprising properties of the SPOs, are determined for arbitrary charge in [17]. The difference between SPO and single-trace operators is crucial for bootstrapping correctly higher genus corrections [16].

The amplitude factor $\mathcal{A}_{\vec{p}}$ is only a function of the conformal cross-ratios $U, V$, and of the R-charge cross ratios $\tilde{U}$ and $\tilde{V}$. We sometimes refer to these cross ratios as AdS and Sphere cross-ratios respectively. The amplitude $\mathcal{A}_{\vec{p}}$ is usually written as a double integral [5] and a double sum [10, 18]: the integral is a Mellin transform, i.e. a Fourier transform with respect to the (logarithm of the) space-time cross-ratios $U, V$. The double sum is a discrete Mellin transform w.r.t. the R-charge cross-ratios $\tilde{U}, \tilde{V}$.

For our study of the large $p$ limit, it will be convenient to transform the discrete sum into a double integral a la Sommerfield, and write the amplitude $\mathcal{A}$ as a four-fold integral in which all Mellin variables are treated equally. The result is what we call the $\operatorname{Ad} S_{5} \times S^{5}$ Mellin representation ${ }^{1}$

$$
\mathcal{A}_{\vec{p}}(U, V, \tilde{U}, \tilde{V})=\iint d s d t \iint d \tilde{s} d \tilde{t} U^{s} V^{t} \tilde{U}^{\tilde{s}} \tilde{V}^{\tilde{t}} \times \Gamma_{\otimes} \times \mathcal{M}_{\vec{p}}(s, t, \tilde{s}, \tilde{t})
$$

where now

$$
\begin{aligned}
\Gamma_{\otimes} & =\mathfrak{S} \frac{\Gamma[-s]^{2} \Gamma[-t]^{2} \Gamma[-u]^{2}}{\Gamma[1+\tilde{s}]^{2} \Gamma[1+\tilde{t}]^{2} \Gamma[1+\tilde{u}]^{2}} ; & \mathfrak{S} & =\pi^{2} \frac{(-)^{\tilde{t}}(-)^{\tilde{u}}}{\sin (\pi \tilde{t}) \sin (\pi \tilde{u})} \\
u & \equiv-s-t-(p+2) ; & \tilde{u} & \equiv-\tilde{s}-\tilde{t}+(p-2) .
\end{aligned}
$$

This formula for $\Gamma_{\otimes}$ refers to the balanced configuration $p_{i=1,2,3,4}=p$. The general case of unequal charges is discussed in appendix A.2. The symbol $\Gamma_{\otimes}$ stresses that this expression contains gamma functions for the product space $A d S_{5} \times S^{5}{ }^{2}$

\footnotetext{
${ }^{1}$ The contour of integration is a standard Mellin-Barnes contour, i.e. a straight line parallel to the imaginary axis separating left poles from right poles, in all complex planes, $s, t, \tilde{s}$ and $\tilde{t}$.

${ }^{2}$ Notice also that the sphere part contains the tree level normalisation found in [15].
} 
When the external charges are large, poles to the left of the original contour are pushed away and we can move the contour to a region where the integration variables are also large. The arguments in $\Gamma_{\otimes}$ then become large and we can replace each gamma function by its Stirling asymptotics

$$
\Gamma(x) \simeq \sqrt{2 \pi} e^{-x} x^{x-\frac{1}{2}} .
$$

Collecting the various exponential contributions we find a simple classical action $S_{\mathrm{cl}}$, and perform the integrations by saddle point. We thus get

$$
\lim _{p \rightarrow \infty} \mathcal{A}_{\vec{p}}(U, V, \tilde{U}, \tilde{V})=\left[\exp \left(-p S_{\mathrm{cl}}\right) \times\left|\operatorname{det}\left(\operatorname{Hessian}\left(S_{\mathrm{cl}}\right)\right)\right|^{-\frac{1}{2}} \times \mathcal{M}_{\vec{p}}\right]_{s_{\mathrm{cl}}, t_{\mathrm{cl}}, \tilde{s}_{\mathrm{cl}}, \tilde{t}_{\mathrm{cl}}}
$$

where the r.h.s. is evaluated at the saddle point $s_{\mathrm{cl}}, t_{\mathrm{cl}}, \tilde{s}_{\mathrm{cl}}, \tilde{t}_{\mathrm{cl}}$. The saddle point is fixed in terms of the space-time and R-charge cross-ratios by extremizing the classical action. On the $A d S_{5}$ we find

$$
-s_{\mathrm{cl}}=p \frac{\sqrt{U}}{1+\sqrt{U}+\sqrt{V}} ; \quad-t_{\mathrm{cl}}=p \frac{\sqrt{V}}{1+\sqrt{U}+\sqrt{V}}
$$

and on the $S^{5}$

$$
\tilde{s}_{\mathrm{cl}}=p \frac{\sqrt{\tilde{U}}}{1+\sqrt{\tilde{U}}+\sqrt{\tilde{V}}} ; \quad \tilde{t}_{\mathrm{cl}}=p \frac{\sqrt{\tilde{V}}}{1+\sqrt{\tilde{U}}+\sqrt{\tilde{V}}} .
$$

The large $p$ limit of the four-point correlator is thus given by the simple formula

$$
\lim _{p \rightarrow \infty} \mathcal{A}_{\vec{p}}(U, V, \tilde{U}, \tilde{V})=\frac{4 \pi^{4}}{(U V \tilde{U} \tilde{V})^{\frac{1}{4}}} \frac{(1+\sqrt{\tilde{U}}+\sqrt{\tilde{V}})^{2(p-2)+\frac{3}{2}}}{(1+\sqrt{U}+\sqrt{V})^{2(p+2)-\frac{3}{2}}} \times\left(p^{4} \lim _{p \rightarrow \infty} \mathcal{M}_{\vec{p}}\left(s_{\mathrm{cl}}, t_{\mathrm{cl}}, \tilde{s}_{\mathrm{cl}}, \tilde{t}_{\mathrm{cl}}\right)\right) .
$$

The combination of the $A d S_{5} \times S^{5}$ saddle is neat. Some terms of the form $e^{ \pm 2 p} p^{\mp 2 p}$ present separately on the factorised $A d S_{5}$ and $S^{5}$ saddles cancel out, and the functions of the cross ratios combine nicely (see appendix B for more details.) We are assuming that at each order in perturbation theory, which here means both $1 / N^{2}$ and $\lambda^{-1 / 2}$, the Mellin amplitude does not exponentiate for large values of the variables, and therefore does not shift the saddle point.

Provided $p \ll \lambda^{1 / 2}$ we expect our computation to reproduce the behaviour of four strings propagating as point-like geodesics in $A d S_{5} \times S^{5}$ until a small interaction region [1$3,5-8,23]$. We will provide further evidence for this picture in section 2 where we will map the first factor in (1.11) to the contribution of four heavy geodesics propagating in the bulk. At the intersection point the strings would now scatter as in flat space. This picture then predicts that $\mathcal{M}_{\vec{p}}\left(s_{\mathrm{cl}}, t_{\mathrm{cl}}, \tilde{s}_{\mathrm{cl}}, \tilde{t}_{\mathrm{cl}}\right)$ should be further related to a scattering amplitude with a precise identification of Mandelstam invariants.

Indeed, the four variable function $\mathcal{M}_{\vec{p}}\left(s_{\mathrm{cl}}, t_{\mathrm{cl}}, \tilde{s}_{\mathrm{cl}}, \tilde{t}_{\mathrm{cl}}\right)$ in the large $p$ limit reduces to a special two-variable function: the ten-dimensional flat space S-matrix of IIB string theory. This is our next non trivial result:

$$
\lim _{p \rightarrow \infty} \mathcal{M}_{\vec{p}}^{A d S_{5} \times S^{5}}\left(s_{\mathrm{cl}}, t_{\mathrm{cl}}, \tilde{s}_{\mathrm{cl}}, \tilde{t}_{\mathrm{cl}} ; \frac{1}{\sqrt{\lambda}} ; \frac{1}{N^{2}}\right)=\frac{1}{\mathbf{s}_{\mathrm{cl}} \mathbf{t}_{\mathrm{cl}} \mathbf{u}_{\mathrm{cl}}} \mathcal{S}^{\text {flat }}\left(\frac{4 \Sigma}{R^{2}} \mathbf{s}_{\mathrm{cl}}, \frac{4 \Sigma}{R^{2}} \mathbf{t}_{\mathrm{cl}} ; \alpha^{\prime} ; g_{s}\right)
$$


where $\Sigma=\frac{1}{2}\left(p_{1}+p_{2}+p_{3}+p_{4}\right), R^{4}=4 \pi g_{s} N \alpha^{\prime 2}$ is the radius, $\lambda=4 \pi g_{s} N$ is the 't Hooft coupling, and the bold font variables are

$$
\mathbf{s}_{\mathrm{cl}}=s_{\mathrm{cl}}+\tilde{s}_{\mathrm{cl}} ; \quad \mathbf{t}_{\mathrm{cl}}=t_{\mathrm{cl}}+\tilde{t}_{\mathrm{cl}} ; \quad \mathbf{s}_{\mathrm{cl}}+\mathbf{t}_{\mathrm{cl}}+\mathbf{u}_{\mathrm{cl}}=0
$$

The connection between the $A d S_{5} \times S^{5}$ Mellin amplitude and the ten-dimensional flat space amplitude here is complete, and depends on all variables $s, t, \tilde{s}, \tilde{t}$ and $p$. Notice that being the S-matrix defined in terms of the Mandelstam invariants, here we have found a non trivial identification of those with geometric data, i.e. a function of the cross ratios, and we have also found a proper identification of the effective coupling for the low energy approximation. In particular, since $\Sigma \mathbf{s}_{\mathrm{cl}} \sim p^{2}$, as far as $\alpha^{\prime}$ goes, the effective couplings is

$$
\frac{p^{2} \alpha^{\prime}}{R^{2}}=\frac{p^{2}}{\sqrt{\lambda}}
$$

The validity of the low energy expansion is precisely the distinction between SUGRA, short strings and big strings that we made in (1.2).

The compatibility of the $A d S_{5} \times S^{5}$ amplitude at fixed $p$, with the ten-dimensional flat space amplitudes in the large $p$ limit, gives us with a new set of optimal constraints for bootstrapping correlators at fixed $p$. Firstly, the uplift of (1.13) to bold font $A d S_{5} \times S^{5}$ variables is dictated by the hidden conformal symmetry at tree level [20], and reads

$$
\mathbf{s}=s+\tilde{s} ; \quad \mathbf{t}=t+\tilde{t} ; \quad \mathbf{s}+\mathbf{t}+\mathbf{u}=-4 .
$$

In particular, the constraint on the r.h.s. goes over the flat space $\mathbf{s}_{\mathrm{cl}}+\mathbf{t}_{\mathrm{cl}}+\mathbf{u}_{\mathrm{cl}}=0$ in the large $p$ limit, since the bold font variables become very large. Secondly, even though $\mathcal{M}_{\vec{p}}(s, t, \tilde{s}, \tilde{t})$ depends on all variables separately, it does it in a particular way. Schematically, we expect

$$
\mathcal{M}_{\vec{p}}^{\ell \text {-loop }} \sim \sum \operatorname{Transcendetal}(\mathbf{s}, \mathbf{t}) \times \operatorname{Rational}_{\vec{p}}(s, t, \tilde{s}, \tilde{t})
$$

where the Transcendetal functions only depend on the bold font variables. These are meromorphic functions in the complex plane such that poles are in correspondence with the OPE. In addition, we now find that these transcendental functions have to asymptote those appearing in the ten-dimensional flat space amplitude [19]. Moreover, at each order in the $\alpha^{\prime}$ expansion of the loop amplitude, the rational functions in $s, t, \tilde{s}, \tilde{t}, \vec{p}$, acquire a stratification under the large $p$ scaling, since this is the natural scaling of the saddle point in (1.9)-(1.10).

We will demonstrate our claims by showing that the large $p$ limit of the $A d S_{5} \times S^{5}$ Virasoro-Shapiro amplitude equals the flat space Virasoro-Shapiro amplitude, written in the variables $\mathbf{s}, \mathbf{t}, \mathbf{u}$, and that the subleading in $p$ contributions are polynomials of lower degree in $s, t, \tilde{s}, \tilde{t}, \vec{p}$, again stratified in powers of $p$. Then, we will uncover a novel Mellin representation at one-loop which encompasses the position space correlators constructed in [16]. Our organisation of the amplitude is thus very natural, and in a sense complements a different approach involving infinite series [41-43], where the transcendental structure of the amplitude is less manifest. 


\section{Intuition from geodesics}

In this section we explain the bulk AdS/CFT picture underlying our large $p$ limit. In particular, the relation between the position space correlator and its Mellin amplitude, given in (1.11), and also for the relation between the Mellin amplitude and the flat space S-matrix, given in (1.12).

Let us begin by interpreting the prefactor,

$$
\text { prefactor }=\frac{4 \pi^{4}}{(U V \tilde{U} \tilde{V})^{\frac{1}{4}}} \frac{(1+\sqrt{\tilde{U}}+\sqrt{\tilde{V}})^{+\frac{3}{2}}}{(1+\sqrt{U}+\sqrt{V})^{-\frac{3}{2}}} \times \underbrace{\left(\frac{1+\sqrt{\tilde{U}}+\sqrt{\tilde{V}}}{1+\sqrt{U}+\sqrt{V}}\right)^{2 p}}_{\text {leading exponential factor }}
$$

which we encountered ${ }^{3}$ in (1.11) as arising from the propagation of four very energetic particles all the way to a common interaction region in $A d S_{5} \times S^{5}$. The idea of our interpretation is similar to that of a three point correlator of three heavy short string states, obtained by Minahan [23]. In our case, the four operators create four particles at the boundary of $A d S_{5} \times S^{5}$, which will meet at a point $(P, \tilde{P})$ in the bulk, whose location we integrate over as in

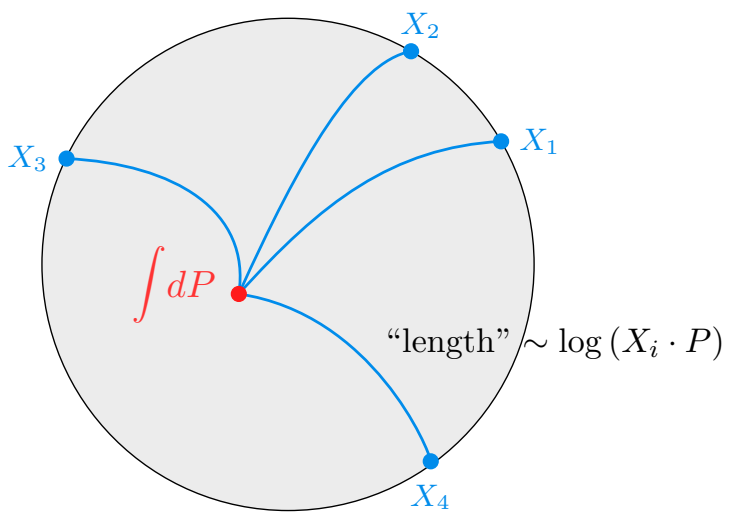

The propagation factor is given by the product of four bulk-to-boundary propagators. To specify those, we will use embedding coordinates. For $A d S_{5}$ we consider the hyperboloid, therefore $P \cdot P=-R^{2}$ is a bulk point, and $X_{i} \cdot X_{i}=0$ lies on the conformal boundary. For the sphere $\tilde{P} \cdot \tilde{P}=+R^{2}$ belongs to $S^{5}$, but $Y_{i}$ is the null six-dimensional polarisation vector on which the $\mathrm{SU}(4) \mathrm{R}$-symmetry acts.

The correlator is thus described by the integral

$$
\int d^{6} P \int d^{6} \tilde{P} \int d \lambda \int d \tilde{\lambda} \prod_{i=1}^{4}\left(\frac{Y_{i} \cdot \tilde{P}}{X_{i} \cdot P}\right)^{p_{i}} e^{-\lambda\left(P \cdot P+R^{2}\right)-\tilde{\lambda}\left(\tilde{P} \cdot \tilde{P}-R^{2}\right)}
$$

where $\lambda$ and $\tilde{\lambda}$ are Lagrange multipliers which enforce $P$ and $\tilde{P}$ to belong to $A d S_{5} \times S^{5}$. For large external charge $p_{i=1,2,3,4}$, the particles are heavy/very energetic and the integration

\footnotetext{
${ }^{3}$ Note that (2.1) is the prefactor in (1.11) if it were not for the $p \pm 2$ shifts. These shifts follows from the superconformal split of the correlator, through the partial non renormalisation theorem, which in (1.3) fixes the space time dependence of kinematics, and therefore fixes the crossing properties of the dynamical amplitude. As far as our bosonic estimates is concerned, we can ignore them without loss of generality.
} 
over the interaction point $(P, \tilde{P})$, is localized by saddle point. ${ }^{4}$ In sum, we should extremize the classical action $S_{\mathrm{cl}}=S_{A d S_{5}}-S_{S^{5}}$ where

$$
S_{A d S_{5}}=\sum_{i=1}^{4} p_{i} \log \left(X_{i} \cdot P\right)-\lambda\left(P \cdot P+R^{2}\right)
$$

and a similar expression holds for the sphere part, which can be obtained by the substitution $X_{i} \rightarrow Y_{i}, P \rightarrow \tilde{P}, \lambda \rightarrow \tilde{\lambda}$, and $R \rightarrow i R$. Notice that the first term (2.4) is indeed the sum of geodesic lengths of four geodesics joining the boundary points $X_{i}$ with the interaction point $P$, as the picture above suggested.

Consider first the eqm obtained by varying with respect to $P$,

$$
\frac{\partial S_{A d S_{5}}}{\partial P_{A}}=0 \quad \text { iff } \quad \sum_{i} p_{i} \frac{X_{i}^{A}}{X_{i} \cdot P}-2 \lambda P^{A}=0 .
$$

Dotting with $P$ leads to $\lambda R^{2}=-\frac{1}{2} \sum_{i} p_{i}$. Dotting with $X_{j}$ yields a set of four equations for the four variables $X_{i} \cdot P$,

$$
\sum_{i \neq j} p_{i} \frac{X_{i} \cdot X_{j}}{X_{i} \cdot P}=-\frac{\left(p_{1}+p_{2}+p_{3}+p_{4}\right)}{R^{2}} X_{j} \cdot P .
$$

For equal charges, we can solve these easily and we find

$$
\begin{aligned}
X_{1} \cdot P & =f \times\left[\frac{\left(X_{1} \cdot X_{2}\right)\left(X_{1} \cdot X_{3}\right)\left(X_{1} \cdot X_{4}\right)}{\left(X_{2} \cdot X_{3}\right)\left(X_{2} \cdot X_{4}\right)\left(X_{3} \cdot X_{4}\right)}\right]^{\frac{1}{4}} \\
f & =\left[\frac{R^{2}}{4}\left(\sqrt{X_{3} \cdot X_{2}} \sqrt{X_{4} \cdot X_{1}}+\sqrt{X_{3} \cdot X_{1}} \sqrt{X_{4} \cdot X_{2}}+\sqrt{X_{2} \cdot X_{1}} \sqrt{X_{4} \cdot X_{3}}\right)\right]^{\frac{1}{2}}
\end{aligned}
$$

with similar expressions for the other $X_{i} \cdot P$. All of them have $f$ as a common factor, and the combinatorics w.r.t. the index $i$ is simple to guess. Now we plug this solution into the action to estimate its leading exponential behaviour. We find a remarkably simple formula

$$
S_{A d S_{5}}^{\text {classical }}=p \log \left(\prod_{i=1}^{4} X_{i} \cdot P\right)=-p \log f^{4} .
$$

If we also factor out $p \log \left(X_{1} \cdot X_{3}\right)\left(X_{2} \cdot X_{4}\right)$ we nicely reproduce the expected coordinate prefactor in the kinematics factor sitting in front of the amplitude plus a cross-ratio dependent expression:

$$
e^{S_{A d S_{5}}^{\text {classical }}}=\frac{1}{\left(X_{1} \cdot X_{3}\right)^{p}\left(X_{2} \cdot X_{4}\right)^{p}} \frac{R^{4}}{16}(1+\sqrt{U}+\sqrt{V})^{-2 p} .
$$

Adding the sphere simply completes this expression in the obvious way, and cancel the radius dependence. Finally

$$
e^{S_{A d S_{5}}^{\text {classical }}-S_{\text {Sphere }}^{\text {classical }}}=\frac{\left(Y_{1} \cdot Y_{3}\right)^{p}\left(Y_{2} \cdot Y_{4}\right)^{p}}{\left(X_{1} \cdot X_{3}\right)^{p}\left(X_{2} \cdot X_{4}\right)^{p}}\left(\frac{1+\sqrt{\tilde{U}}+\sqrt{\tilde{V}}}{1+\sqrt{U}+\sqrt{V}}\right)^{2 p} .
$$

We have thus reproduced the leading exponential factor in (2.1).

\footnotetext{
${ }^{4}$ In unpublished work from 2017, while studying the flat space limit of general multi-point functions of gapped QFT's in AdS, Shota Komatsu has solved similar problems; see in particular [9]. We thank Shota for several insightful discussions on this point.
} 
Let us turn now to the non-exponential factor in (2.1). In the saddle point approximation, that we just found above, we expect the non-exponential factor to arise from integrating out quadratic fluctuations. For the $A d S_{5}$ part, the quadratic action around the saddle can be written as

$$
\frac{\partial^{2}}{\partial P_{A} \partial P_{B}} S_{A d S_{5}}=p \sum_{i=1}^{4}\left[-\frac{X_{i}^{A} X_{i}^{B}}{\left(X_{i} \cdot P\right)^{2}}+\frac{1}{R^{2}} \delta_{A B}\right] .
$$

To compute the determinant is convenient to go to the basis of the $X_{i}$. In total, the gaussian integration produces

$$
\frac{(2 \pi)^{2}}{p^{2}} \sqrt{\frac{\operatorname{det}_{j, k}\left[X_{j} \cdot X_{k}\right]}{\operatorname{det}_{j, k}\left[\sum_{i=1}^{4} \frac{X_{j} \cdot X_{i} X_{i} \cdot X_{k}}{\left(X_{i} \cdot P\right)^{2}}+X_{j} \cdot X_{k}\right]}}=\frac{\pi^{2} R^{4}}{16 p^{2}} \frac{(1+\sqrt{U}+\sqrt{V})^{\frac{3}{2}}}{(U V)^{\frac{1}{4}}} .
$$

where $\operatorname{det}\left[X_{j} \cdot X_{k}\right]$ takes into account the non-orthonormality of the basis we are using. Combining with the sphere contribution we beautifully match the full prefactor in (2.1) up to a simple numerical factor. ${ }^{5}$

Now we would like to explain with a simple argument our second claim in (1.12), namely, how the relation between the large $p$ limit of the Mellin amplitude and the flat space S-matrix comes about in our picture. The idea - nicely described in [23] — is to measure the slope of each geodesic at the intersection point $(P, \tilde{P})$, and extract the corresponding momenta as it enters the interaction region. At this point, note that the saddle point equations for the two factors of $A d S_{5}$ and $S^{5}$ are nothing but "momentum conservation" in the corresponding embedding spaces, if we define

$$
\begin{array}{ll}
M_{i}^{A} \equiv p_{i}\left(\frac{X_{i}^{A}}{X_{i} \cdot P}+\frac{P^{A}}{R^{2}}\right) ; & \sum_{i} M_{i}^{A}=0 \\
\tilde{M}_{i}^{\tilde{A}} \equiv p_{i}\left(\frac{Y_{i}^{\tilde{A}}}{Y_{i} \cdot \tilde{P}}-\frac{\tilde{P}^{\tilde{A}}}{R^{2}}\right) ; & \sum_{i} \tilde{M}_{i}^{\tilde{A}}=0 .
\end{array}
$$

Notice that $M_{i}^{A}$ is orthogonal to $P$ and $M_{i}^{\tilde{A}}$ is orthogonal to $\tilde{P}$. Moreover

$$
M_{i} \cdot M_{i}=+\frac{p_{i}^{2}}{R^{2}} ; \quad \tilde{M}_{i} \cdot \tilde{M}_{i}=-\frac{p_{i}^{2}}{R^{2}} .
$$

We can then assemble four twelve-dimensional vectors,

$$
K_{i}=\left(M_{i}^{A}, \tilde{M}_{i}^{\tilde{A}}\right) ; \quad i=1,2,3,4
$$

which by constructions are null and tangent to the $10 \mathrm{~d}$ geodesics at the intersection point. They are the 10d Lorentzian momenta. By explicit computation we find ${ }^{6}$

$$
\left.2 K_{1} \cdot K_{2}\right|_{p_{i}=p}=\frac{8 p}{R^{2}}\left[\frac{p \sqrt{U}}{\sqrt{U}+\sqrt{V}+1}-\frac{p \sqrt{\tilde{U}}}{\sqrt{\tilde{U}}+\sqrt{\tilde{V}}+1}\right]=\left.\frac{4 \Sigma}{R^{2}} \mathbf{s}_{\mathrm{cl}}\right|_{p_{i}=p}
$$

\footnotetext{
${ }^{5}$ The numerical factor is $R^{8} / 2^{6} /(2 p)^{4}$.

${ }^{6}$ We are using a convention where $2 K_{1} \cdot K_{2}=-\left(k_{1}+k_{2}\right)^{2}$ when going from embedding to 10d Lorentzian momenta, see e.g. (13) in [5].
} 
and similarly for the others thus perfectly reinforcing the identifications of the previous section. Relation (2.11) is a very important dictionary type relation; it gives us the link between physical Mandelstam invariants, Mellin variables and space-time cross-ratios.

We can now go back to our picture (2.2) and turn on interactions, locally at the intersection point. This should simply decorate the integrand with the $10 \mathrm{~d}$ flat space Smatrix. From the identification of physical Mandelstam invariants and Mellin variables given above, we can then argue that the large $p$ limit of the $A d S_{5} \times S^{5}$ Mellin amplitude is expected to behave as anticipated in (1.12) above.

In the next two sections we demonstrate this relation in a very precise way. Firstly by looking at the genus zero amplitude, and then by looking at one-loop supergravity.

\section{Genus zero amplitude}

\subsection{Tree level SUGRA}

The tree level Mellin amplitude for arbitrary charges was discovered in the seminal paper of $[10,18]$, and it was shown to possess a hidden ten dimensional conformal symmetry by Caron-Huot and Trinh in [20]. This hidden symmetry explains the beautiful pattern of anomalous dimensions uncovered in [15]. In our notation, the tree level Mellin amplitude in (1.5) for arbitrary charges $\vec{p}$ looks extremely simple. It is just ${ }^{7}$

$$
\mathcal{M}_{p_{1} p_{2} p_{3} p_{4}}^{(1)}(s, t, \tilde{s}, \tilde{t})=-\frac{1}{(1+\mathbf{s})(1+\mathbf{t})(1+\mathbf{u})},
$$

where $\mathbf{s}+\mathbf{t}+\mathbf{u}=-4$. The hidden conformal symmetry is beautifully manifest in the bold font variables, i.e. the four variable function $\mathcal{M}_{\vec{p}}^{(1)}(s, t, \tilde{s}, \tilde{t})$ is shown here to depend only on $\mathbf{s}, \mathbf{t}$ and $\mathbf{u}$.

We can now start from the flat space limit a la Penedones, in which $s$ and $t$ are large but $\tilde{s}$ and $\tilde{t}$ are kept finite. As a result

$$
\lim _{s, t, \rightarrow \infty} \mathcal{M}_{p_{1} p_{2} p_{3} p_{4}}^{(1)}(s, t, \tilde{s}, \tilde{t}) \rightarrow \frac{1}{s t u}
$$

where $u$ participates in the limit as $u \rightarrow-s-t$. This limit is very asymmetric from the point of view of $A d S_{5} \times S^{5}$, because in reality all four variables $s, t$, and $\tilde{s}, \tilde{t}$ are on equal footing in the $A d S_{5} \times S^{5}$ Mellin amplitude. In fact, the flat space limit a la Penedones and the large $p$ limit are similar, in the sense that in both cases one considers large Mellin variables, but the first one 'forgets' about the sphere, because $\tilde{s}$ and $\tilde{t}$ are kept finite. If we want to restore the sphere what we should do is to covariantise (3.2) and write

$$
\left.\lim _{p \rightarrow \infty} \mathcal{M}_{p_{1} p_{2} p_{3} p_{4}}^{(1)}(s, t, \tilde{s}, \tilde{t}) \rightarrow\left(\frac{1}{\mathbf{s t u}}\right)\right|_{\mathbf{s}=\mathbf{s}_{\mathrm{cl}}, \mathbf{t}=\mathbf{t}_{\mathrm{cl}}, \mathbf{u}=\mathbf{u}_{\mathrm{cl}}} .
$$

The r.h.s. of the formula above is non trivial in two ways: we first covariantise the flat space limit in (3.2) with $\mathbf{s}, \mathbf{t}$ and $\mathbf{u}=-\mathbf{s}-\mathbf{t}-4$, and then localise the amplitude on the flat

\footnotetext{
${ }^{7}$ The bold font variables are still given by (1.15) with $p \rightarrow p_{3}$, while $\Gamma_{\otimes}$ upgrades to (A.18).
} 
space saddle point $\mathbf{s}_{\mathrm{cl}}+\mathbf{t}_{\mathrm{cl}}+\mathbf{u}_{\mathrm{cl}}=0$. This aspect of our formalism is tied with the hidden symmetry [20], which partly born out from the idea of relating the $p_{1} p_{2} p_{3} p_{4}$ amplitude to the simplest 2222 correlator. In particular, the variables $\mathbf{s}, \mathbf{t}, \mathbf{u}$, satisfy the same constraint as the $A d S_{5}$ Mellin variables $s, t, u$, for the 2222 correlator, and this is why we can always covariantise as above. ${ }^{8}$ The $A d S_{5} \times S^{5}$ Mellin representation allows us to make manifest both the covariantisation for large bold font Mellin variables, and the hidden symmetry of the correlator. We shall see that the covariatisation principle holds always, both in the full genus zero amplitude, and at one-loop.

\section{2 $\quad$ Large $p$ limit $=10 \mathrm{~d}$ Virasoro-Shapiro}

In this section we consider $\alpha^{\prime}$ genus zero corrections to tree level supergravity.

Starting from the flat space formula of Penedones [5], (which is valid for fixed $s u(4)$ channel) we obtain the integral relation

$$
\mathcal{M}(s, t)=C \int_{0}^{\infty} d z z^{\Sigma-\frac{d}{2}+3} e^{-z} \mathcal{A}_{\mathrm{VS}}^{\mathrm{flat}}\left(\frac{4 z}{R^{2}} s, \frac{4 z}{R^{2}} t\right) ; \quad s, t \gg 1
$$

where $C$ is a normalisation and $\Sigma=\frac{1}{2} \sum_{i} p_{i}$. The $\alpha^{\prime}$ expansion of the VS amplitude is polynomial, thus one approach would be to make a polynomial ansatz for $\mathcal{M}(s, t)$, and match order by order the $\alpha^{\prime}$ expansion of the r.h.s. This computation boils down to $\Gamma$ function integrals multiplying the expansion of the VS amplitude, and thus fixes the leading polynomial term of the $A d S_{5} \times S^{5}$ amplitude. We have done this in appendix C.2, where we also explain in more details how the covariantisation principle works. Here instead we will take a quicker route which leads to the same result.

Note that (3.4) defines a Mellin amplitude which is also a function of $\vec{p}$, because even though the flat space VS amplitude has no knowledge of $\vec{p}$, the remaining integrand in $z$ depends on $\Sigma$. Then, by taking $p_{i} \rightarrow \infty$ the integral localises on a saddle point. The effective action is $\Sigma \log z-z$ and the saddle is $z=\Sigma$. This saddle point evaluation is almost like our master formula (1.12), but for the fact that we forgot the sphere. However, we learned in the previous section how to restore the dependence on $\tilde{s}$ and $\tilde{t}$. This is as simple as covariantising the result in $s$ and $t$, to a function of $\mathbf{s}$ and $\mathbf{t}$.

Summarising, the large $p$ limit of the genus zero $A d S_{5} \times S^{5}$ amplitude is the flat space VS amplitude evaluated at $4 \Sigma \mathbf{s}_{\mathrm{cl}} / R^{2}$ and $4 \Sigma \mathbf{t}_{\mathrm{cl}} / R^{2}$, which are the covariantised variables appearing in (3.4), with $z=\Sigma$, evaluated at the saddle point since we are taking $p$ large in the first place. This is the same identification of Mandelstam invariants we obtained from our picture of four point-like strings interacting at the bulk point in the previous section.

Putting together our two computations to fix the details, and using the explicit expression for the Virasoro-Shapiro amplitude recalled in appendix C.1, we arrive at the prediction

$$
\left.\lim _{p \rightarrow \infty} \mathcal{M}\left(s_{\mathrm{cl}}, t_{\mathrm{cl}}, \tilde{s}_{\mathrm{cl}}, \tilde{t}_{\mathrm{cl}}\right)\right|_{\text {genus }=0}=\frac{1}{\mathbf{s}_{\mathrm{cl}} \mathbf{t}_{\mathrm{cl}} \mathbf{u}_{\mathrm{cl}}} \frac{\Gamma\left[1-\frac{\Sigma}{\sqrt{\lambda}} \mathbf{s}_{\mathrm{cl}}\right] \Gamma\left[1-\frac{\Sigma}{\sqrt{\lambda}} \mathbf{t}_{\mathrm{cl}}\right] \Gamma\left[1-\frac{\Sigma}{\sqrt{\lambda}} \mathbf{u}_{\mathrm{cl}}\right]}{\Gamma\left[1+\frac{\Sigma}{\sqrt{\lambda}} \mathbf{s}_{\mathrm{cl}}\right] \Gamma\left[1+\frac{\Sigma}{\sqrt{\lambda}} \mathbf{t}_{\mathrm{cl}}\right] \Gamma\left[1+\frac{\Sigma}{\sqrt{\lambda}} \mathbf{u}_{\mathrm{cl}}\right]} .
$$

\footnotetext{
${ }^{8}$ The same conclusion can be reached by using the operators $\widehat{\mathcal{D}}(\vec{p})$ applied to the 2222 amplitude, as in the original argument in [20]. We shall use this formalism later, when we discuss the one-loop amplitudes.
} 
From the resummed amplitude we can appreciate the three different regimes we mentioned in the Introduction. Begin by noticing that the 'coupling' entering the argument of the $\Gamma$ function is

$$
\epsilon=\frac{\Sigma}{\sqrt{\lambda}} ; \quad \Sigma=\frac{p_{1}+p_{2}+p_{3}+p_{4}}{2} ; \quad|\epsilon| \ll 1
$$

where $|\epsilon| \ll 1$ defines our setting. At the saddle point both $p_{i=1,2,3,4}$ and the Mellin variables are taken to be large in the same way. For concreteness, let's say $\mathbf{s} / p$ fixed with $p \rightarrow \infty$. This ratio is a function which we might call geometry, since it depend only on the cross ratios. The effective coupling controlling the behaviour of the amplitude is thus

$$
\epsilon_{\mathrm{eff}}=\frac{p^{2}}{\sqrt{\lambda}}
$$

and the three regimes are

\begin{tabular}{|c|c|c|}
\hline$p \ll \lambda^{1 / 4}$ & SUGRA & $\Gamma[1 \pm \epsilon \mathbf{s}] \rightarrow 1$ \\
\hline$p / \lambda^{1 / 4}=O(1)$ & short massive strings & $\Gamma[1 \pm \epsilon \mathbf{s}]$ held fixed \\
\hline$\lambda^{1 / 4} \ll p \ll \lambda^{1 / 2}$ & flat classical strings & $\Gamma[1 \pm \epsilon \mathbf{s}] \rightarrow \exp [ \pm \epsilon \mathbf{s} \log \mathbf{s}]$ \\
\hline
\end{tabular}

The SUGRA regime is improved by taking $p / \lambda^{1 / 4}=x$ fixed with $x \ll 1$. This corresponds to taking into account higher derivative corrections to the SUGRA action, coming from string theory, which we see here as an expansion in small $\epsilon \mathbf{s}$. Instead, in the regime of flat classical strings we cross to the high-energy regime, where $\epsilon \mathbf{S}$ is large, the amplitude exponentiates

$$
\begin{aligned}
& \left.\lim _{p \rightarrow \infty} \mathcal{M}\left(s_{\mathrm{cl}}, t_{\mathrm{cl}}, \tilde{s}_{\mathrm{cl}}, \tilde{t}_{\mathrm{cl}}\right)\right|_{\text {genus }=0}= \\
& \quad \frac{1}{\mathbf{s}_{\mathrm{cl}} \mathbf{t}_{\mathrm{cl}} \mathbf{u}_{\mathrm{cl}}} \exp \left[-\epsilon\left(\mathbf{s}_{\mathrm{cl}} \log \left(-\mathbf{s}_{\mathrm{cl}}^{2}\right)+\mathbf{t}_{\mathrm{cl}} \log \left(-\mathbf{t}_{\mathrm{cl}}^{2}\right)+\mathbf{u}_{\mathrm{cl}} \log \left(-\mathbf{u}_{\mathrm{cl}}^{2}\right)\right)\right](1+\ldots) .
\end{aligned}
$$

The exponential contribution is precisely the Gross-Mende amplitude [24]. This has the interpretation of the saddle point action for a minimal area surface in flat space contributing to the four-point scattering amplitude. If before we had two decoupled geodesic saddles, separately on $A d S_{5}$ and $S^{5}$, coming from $\Gamma_{\otimes}$, the effect of reaching a transition to a stringylike object is to couple the two with flat space interactions mediated by the Gross-Mende amplitude. Still, as long as $\epsilon$ is small, even a classical string such as that in Gross-Mende is still only probing a small portion of space around the bulk point where the dynamics is like flat space, see figure 1. If we further increase $p \rightarrow \sqrt{\lambda}, \epsilon$ is no longer small and our approximation breaks down. Physically it is clear why: we would still be describing strings fluctuations in a flat space approximation, rather than the full $A d S_{5} \times S^{5}$. We will comment more about the transition from flat to big strings in $A d S_{5} \times S^{5}$ in the discussion section 5 . 


\subsection{Towards the full amplitude: $\zeta_{3}$ and $\zeta_{5}$}

The $A d S_{5} \times S^{5}$ Virasoro-Shapiro amplitude is there, waiting to be discovered, and only the first two terms in the $\alpha^{\prime}$ expansion are known in full generality [48, 49]. These have been constructed by complementing the results of $[40,41]$, which used the flat space limit a la Penedones, with the information coming from the unmixing problem [13], pushed to order $\alpha^{\prime 3}$ and $\alpha^{\prime 5}$, and localisation results from [44-47].

At each order in the $\alpha^{\prime}$ expansion, the $A d S_{5} \times S^{5}$ Virasoro-Shapiro amplitude is a polynomial in the Mellin variables, but differently from its flat space limit, it is a sum of polynomials of different degree. The large $p$ limit suggests how to organise this sum. The idea is simply to stratify the amplitude according to the scaling

$$
s \rightarrow p s ; \quad t \rightarrow p t ; \quad \tilde{s} \rightarrow p s ; \quad \tilde{t} \rightarrow p \tilde{t} ; \quad p \rightarrow \infty
$$

which is indeed the scaling of the saddle point solutions (1.9) and (1.10). Then, each stratum of the amplitude, written in the four fold representation, must be a function of crossing symmetric polynomials in the letters

$$
\mathbf{s}, \mathbf{t}, \mathbf{u} ; \quad \tilde{s}, \tilde{t}, \tilde{u} \equiv-\tilde{s}-\tilde{t}+p_{3}-2 ; \quad p_{1}, p_{2}, p_{3}, p_{4} .
$$

To demonstrate our observation we will now rewrite the results of $[48,49]$ according to our discussion. We work directly with the most general case of arbitrary external charges, and find that

$$
\begin{aligned}
\zeta_{3}^{-1} \mathcal{V}^{(1,0)}= & (\Sigma-1)_{3} \times 2 \\
\zeta_{5}^{-1} \mathcal{V}^{(1,2)}= & (\Sigma-1)_{5}\left(\mathbf{s}^{2}+\mathbf{t}^{2}+\mathbf{u}^{2}\right) \\
& +(\Sigma-1)_{4}\left(-10(\tilde{s} \mathbf{s}+\tilde{t} \mathbf{t}+\tilde{u} \mathbf{u})-5\left(c_{s} \mathbf{s}+c_{t} \mathbf{t}+c_{u} \mathbf{u}\right)\right) \\
& +(\Sigma-1)_{3}\left(+20\left(\tilde{s}^{2}+\tilde{t}^{2}+\tilde{u}^{2}\right)+\frac{5}{2}\left(c_{s}^{2}+c_{t}^{2}+c_{u}^{2}\right)+20\left(\tilde{s} c_{s}+\tilde{t} c_{t}+\tilde{u} c_{u}\right)\right) \\
& +(\Sigma-1)_{3}\left(+\frac{33}{2}-\frac{27}{2} \Sigma^{2}\right) .
\end{aligned}
$$

For convenience of the reader we repeat

$c_{s}=\frac{p_{1}+p_{2}-p_{3}-p_{4}}{2} ; \quad c_{t}=\frac{p_{1}+p_{4}-p_{2}-p_{3}}{2} ; \quad c_{u}=\frac{p_{2}+p_{4}-p_{3}-p_{1}}{2} ; \quad \Sigma=\frac{p_{1}+p_{2}+p_{3}+p_{4}}{2}$.

Let us focus on the lines (3.11) and (3.12) first. The Pochhammers in $\Sigma$ come from the (inverse) $\Gamma$ function integral built in the flat space limit a la Penedones, (as we show in (C.8) in appendix C.2). In particular, from the latter one finds the leading terms $\zeta_{3}(\Sigma-1)_{3}$ and $\zeta_{5}(\Sigma-1)_{5}\left(s^{2}+t^{2}+u^{2}\right)$. This was the starting point in $[48,49]$. We see now that their result secretly covariantises, as we argued in the previous section. Notice that away from the saddle point solution, the covariantisation is in terms of bold font variables such that $\mathbf{s}+\mathbf{t}+\mathbf{u}=-4$. Taking the large $p$ limit amounts to turn the Pochhammers into powers, 
thus in the large $p$ limit we find the flat space VS amplitude in $\mathbf{s}$ and $\mathbf{t}$, expanded to the given order. For example,

$$
\lim _{p \rightarrow \infty} \mathcal{V}^{(1,2)}\left(s_{\mathrm{cl}}, t_{\mathrm{cl}}, \tilde{s}_{\mathrm{cl}}, \tilde{t}_{\mathrm{cl}}\right)=\zeta_{5} \Sigma^{5}\left(\mathbf{s}_{\mathrm{cl}}^{2}+\mathbf{t}_{\mathrm{cl}}^{2}+\mathbf{u}_{\mathrm{cl}}^{2}\right)
$$

The $\zeta_{5}$ contribution is the first non trivial case where polynomials of lower degree in the Mellin variables are turned on, due to $A d S_{5} \times S^{5}$ effects. The large $p$ stratification is manifest in the polynomials which accompany the various factors $(\Sigma-1)_{k \leq 4}$. Indeed, if we consider the $(\Sigma-1)_{4}$ term, this can only multiply a polynomial which is linear in $\mathbf{s}$ and $\mathbf{t}$ and at most linear in the remaining variables, $\tilde{s} \tilde{t}$ and $p_{i=1,2,3,4}$, otherwise it would contribute to the large $p$ limit. The concrete result from [49] becomes amazingly simple. A similar observation holds for the $(\Sigma-1)_{3}$ contribution. ${ }^{9}$ In addition, the overall degree of $\mathcal{V}^{(1,2)}$ w.r.t. $\tilde{s}$ and $\tilde{t}$ is expected to be at most second order, i.e. it should not exceed the degree of the top term. ${ }^{10}$

\section{One-loop SUGRA}

The next level of computations we can explore with our large $p$ limit is that of one-loop SUGRA. Even though there are no Witten diagrams computations of such kind, a number of four-point one-loop amplitudes, fully consistent with the dual CFT picture, have been bootstrapped in [12-16]. These provide the basis for our explorations as we now explain.

Understanding the anatomy of the four-point one-loop amplitudes goes through the analysis of the spectrum of two-particle operators in $\mathcal{N}=4$ SYM $[15,16]$. Long twoparticle operators are exchanged in the leading logarithmic discontinuity, more generally at any loop order $\ell$, and determine the $\log ^{\ell+1} u$ coefficient function of the full amplitude, in terms of their tree level anomalous dimensions (to the power $\ell+1$ ) and their leading three point couplings with the external single-particle operators. To complete the logarithmic discontinuity into the full one-loop amplitude, the entire two-particle data, both long and protected, is needed. Luckily for us this can all be extracted from the tree level amplitude in combination with $1 / N^{2}$ free theory, and propagated in the one-loop amplitude according to the OPE. In this way the full one-loop amplitude is fixed, up to stringy ambiguities.

In principle all Kaluza Klein one-loop amplitudes are available from the position space algorithm of [16]. However, the large $p$ limit is hard to study in position space. Thus we will have to Mellinize those results. The strategy we follow is to use the hidden conformal symmetry [20] to write the leading logarithmic discontinuity of a generic correlator in one go. Then, we Mellinize the full position space results and take the large $p$ limit.

The consistency of the large $p$ limit with the flat space scattering amplitude suggests from the very beginning that the transcendental content of the one-loop Mellin amplitude at fixed $p$ is made of meromorphic functions which match those of the $10 \mathrm{~d}$ box in the limit. We will show that this pattern is the very much the same as for the position space

\footnotetext{
${ }^{9}$ Localisation only enters to fix the actual value of $\frac{33}{2}-\frac{27}{2} \Sigma^{2}+\frac{5}{2}\left(c_{s}^{2}+c_{t}^{2}+c_{u}^{2}\right)$. We thanks J. Drummond and M. Santagata for discussion on this point.

${ }^{10}$ In the superblock basis this is related to the truncation of the $10 \mathrm{~d}$ spin in the spectrum, i.e. the fact that only rep $[a b a]$ with $a=0,1,2$ appear at this order in the OPE.
} 
amplitude. On one hand, this is the only way position and Mellin space could match precisely, on the other hand this computation is highly non trivial.

Some readers might wish to jump directly to section 4.3 at this point. There we collect the various more technical results obtained in sections 4.1 and 4.2 and we discuss directly the correspondence of the large $p$ limit with the 10d S-matrix.

\subsection{Logarithmic discontinuities}

In the formalism of [20], the $\log ^{2} u$ discontinuity is obtained by acting with an eight-order differential operator, see $\Delta^{(8)}$ in appendix $\mathrm{D}$, on the following prepotential,

$$
\mathcal{P}_{p_{1} p_{2} p_{3} p_{4}}^{\ell}(U, V, \tilde{U}, \tilde{V})=\sum_{T}\left(\frac{\tilde{U}}{U}\right)^{\tilde{s}+2}\left(\frac{\tilde{V}}{V}\right)^{\tilde{t}} \widehat{\mathcal{D}}_{\tilde{s}, \tilde{t}}\left(p_{1} p_{2} p_{3} p_{4}\right) \mathcal{P}_{2222}^{\ell}(U, V) .
$$

By the hidden conformal symmetry the operators $\widehat{\mathcal{D}}_{\tilde{s}, \tilde{t}}\left(p_{1} p_{2} p_{3} p_{4}\right)$ do not depend on the loop order, i.e. they are the same operator at tree level. We have found the closed form expression for these operators: ${ }^{11}$

$$
\begin{aligned}
\widehat{\mathcal{D}}_{\tilde{s}, \tilde{t}}(\vec{p})= & \frac{\left(U \partial_{U}-3-\tilde{s}\right)_{\tilde{s}}}{\tilde{s} !} \frac{\left(U \partial_{U}-3-\tilde{s}-c_{s}\right)_{\tilde{s}+c_{s}}}{(-)^{c_{s}}\left(\tilde{s}+c_{s}\right) !} \times \\
& \frac{\left(V \partial_{V}+1-\tilde{t}\right)_{\tilde{t}}}{\tilde{t} !} \frac{\left(V \partial_{V}+1-\tilde{t}-c_{t}\right)_{\tilde{t}+c_{t}}}{(-)^{c_{t}}\left(\tilde{t}+c_{t}\right) !} \times \frac{\left(U \partial_{U}+V \partial_{V}\right)_{\tilde{u}}}{\tilde{u} !} \frac{\left(U \partial_{U}+V \partial_{V}\right)_{\tilde{u}+c_{u}}}{\left(\tilde{u}+c_{u}\right) !} .
\end{aligned}
$$

The one loop function $\mathcal{P}_{2222}(u, v)$ has a very simple form, given in [20] in position space. (It can be found in appendix D in our notation.) Our construction of the one-loop Mellin amplitudes instead begins with the following Mellin representation for $\mathcal{P}_{2222}$ :

$$
\mathcal{P}_{2222}(U, V)=-\iint d s d t(-U)^{s+4} V^{t} \frac{\Gamma[-s]}{\Gamma[s+1]} \Gamma[-t]^{2} \Gamma[-u]^{2} \times \mathcal{N}_{2222}(s, t)
$$

where $u=-s-t-4$ for this correlator, and the Mellin amplitude is

$$
\begin{aligned}
& \mathcal{N}_{2222}(s, t)= \\
& \quad\left(\psi^{(0)}(-t)-\psi^{(0)}(s+1)\right) \mathcal{T}^{(4)}(s, t)+\left(\psi^{(0)}(-u)-\psi^{(0)}(s+1)\right) \mathcal{T}^{(4)}(s, u)+\mathcal{T}^{(3)}(s, t)
\end{aligned}
$$

with the functions $\mathcal{T}^{(4)}$ and $\mathcal{T}^{(3)}(s, t)=\mathcal{T}^{(3)}(s, u)$ given by ${ }^{12}$

$$
\begin{aligned}
\mathcal{T}^{(4)}(s, t) & =\frac{1}{120} \frac{(t-1) t}{(s+1)(s+2)(s+t+1)(s+t+2)(s+t+3)} \\
\mathcal{T}^{(3)}(s, t) & =\frac{1}{240} \frac{(s+3)}{(s+1)} \frac{16+8(t+u)-4(t-u)^{2}+u t(t+u)-2\left(t^{3}+u^{3}\right)}{(s+1)(s+2)(t+1)(t+2)(u+1)(u+2)} .
\end{aligned}
$$

The contour of integration in (4.3) is a straight line Mellin-Barnes contour. ${ }^{13}$

\footnotetext{
${ }^{11}$ Recall (3.10) for the definition of $\tilde{u}$ and (3.13) for $c_{s}, c_{t}, c_{u}$.

${ }^{12}$ The (top weight) function $\mathcal{T}^{(4)}[s, t]$ is the Mellin transform of $2 \times \mathcal{P}_{2222}^{2,2^{-}} /\left(x_{1}-x_{2}\right)^{7}$. It follows from the Mellin representation of $\left(x_{1}-x_{2}\right)^{-7}$, after including the numerator, monomial by monomial by shifting the contour of integration, as suggested in [16]. This procedure works at any loop order for the top-weight amplitude.

${ }^{13}$ It can be deformed to encircle poles at $s \geq 0$ and $t \geq 0$, so to match the Taylor expansion in position space. Notice there is actually no simple pole at $t=-1$ !, or at $s=-1$.
} 
Turning the operators $\widehat{\mathcal{D}}_{\tilde{s}, \tilde{t}}\left(p_{1} p_{2} p_{3} p_{4}\right)$ into Mellin space, we arrive at the counterpart of the formula (4.1) in Mellin space. This computation, which looks complicated, can actually be done with pencil and paper, and we do so in appendix D. The result is very illuminating

$$
\begin{aligned}
& \mathcal{P}_{p_{1} p_{2} p_{3} p_{4}}(U, V, \tilde{U}, \tilde{V})= \\
& (U \tilde{U})^{2} \iint \tilde{U}^{\tilde{s}} \tilde{V}^{\tilde{t}}\left[(-)^{\max \left(0, c_{s}\right)} \iint(-U)^{s} V^{t}\left[\frac{\sin \left(\pi\left(-s+\max \left(0, c_{s}\right)\right)\right)}{\pi} \Gamma_{\otimes}\right] \mathcal{N}_{2222}(\mathbf{s}, \mathbf{t})\right]
\end{aligned}
$$

where the sin flips one of the two $s$-dependent $\Gamma$ functions in $\Gamma_{\otimes}$, in order to have just simple poles, as it is the case for the leading logarithmic discontinuity. From (4.7) we read off the Mellin amplitude of the prepotential for arbitrary charges,

$$
\mathcal{N}_{p_{1} p_{2} p_{3} p_{4}}(s, t, \tilde{s}, \tilde{t})=\mathcal{N}_{2222}(\mathbf{s}, \mathbf{t})
$$

where the r.h.s. is evaluated precisely at the bold font 10d variables defined in (1.15).

In position space $\mathcal{P}_{p_{1} p_{2} p_{3} p_{4}}$ is simpler than the corresponding leading logarithmic discontinuity, but in Mellin space all the complexity has collapsed to just the $\Gamma_{\otimes}$ factor, and the rest is carried by $\mathcal{N}_{p_{1} p_{2} p_{3} p_{4}}(s, t, \tilde{s}, \tilde{t})=\mathcal{N}_{2222}(\mathbf{s}, \mathbf{t})$, namely the $\mathcal{N}_{2222}(s, t)$ prepotential promoted to be a function of the bold font variables $\mathbf{s}$, and $\mathbf{t}$. The fact that $\mathcal{N}_{p_{1} p_{2} p_{3} p_{4}}$ depends only on bold font variables perhaps was to be expected, since the prepotential is obtained by a "unitarity cut" formula at tree level, and therefore still enjoys the hidden conformal symmetry.

The double discontinuity of $\mathcal{A}_{p_{1} p_{2} p_{3} p_{4}}^{(2)}$ is finally obtained by acting with the eight-

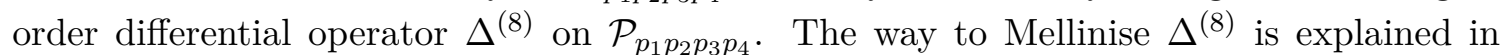
appendix D, roughly what happens is that we write $\Delta^{(8)}=\sum \Omega_{m n \tilde{m} \tilde{n}} U^{m} V^{n} \tilde{U}^{\tilde{m}} \tilde{V}^{\tilde{n}}$, and by shifting the contour we turn the monomials $U^{n} V^{m} \tilde{U}^{i} \tilde{V}^{j}$ into a shift operator. This procedure is straightforward but less illuminating, since it involves some lengthy polynomials in the Mellin variables. Supplementary material is attached to appendix D to help the reader.

\subsection{One-loop Mellin amplitudes}

The total one-loop amplitude for a balanced configuration of charge, $p_{i=1,2,3,4}=p$ has the form

$$
\mathcal{A}_{p p p p}^{(2)}=\sum_{\tilde{s}, \tilde{t}} \iint \frac{\Gamma[-s]^{2} \Gamma[-t]^{2} \Gamma[-u]^{2}}{\Gamma[\tilde{s}+1]^{2} \Gamma[\tilde{t}+1]^{2} \Gamma[\tilde{u}+1]^{2}} U^{s} V^{t} \tilde{U}^{\tilde{s}} \tilde{V}^{\tilde{t}} \underbrace{\left(\mathcal{W}_{p p p p}^{w=4}+\mathcal{W}_{p p p p}^{w=3}+\mathcal{W}_{p p p p}^{w=2}\right)}_{\mathcal{M}_{p p p p}^{(2)}(s, t, \tilde{s}, \tilde{t})} .
$$

It consists of three sub-amplitudes $\mathcal{W}^{w=4,3,2}$ distinguished by the transcendental weight $w$ they first contribute to. Each of these functions can be written as a sum of products of reduced transcendental kernels multiplying rational functions. The full transcendental kernels include of course $\Gamma_{\otimes}$, which we wrote explicitly in (4.9).

We will now introduce the various $\mathcal{W}_{p p p p}^{w=4,3,2}$ and take the large $p$ limit. Doing so we will remark how the organisation of the integrand in (4.9) is in perfect agreement with the position space results of [16]. The case of unequal external charge follows a similar construction, and will be detailed in a future work. 


\subsubsection{Top-weight 4}

The structure of the top-weight 4 integrand is

$$
\mathcal{W}_{p p p p}^{w=4}=\mathcal{K}[\mathbf{s}, \mathbf{t}] \mathcal{W}_{p p p p}^{(4)}(s, t, \tilde{s}, \tilde{t})+\mathcal{K}[\mathbf{s}, \mathbf{u}] \mathcal{W}_{p p p p}^{(4)}(s, u, \tilde{s}, \tilde{u})+\mathcal{K}[\mathbf{u}, \mathbf{t}] \mathcal{W}_{p p p p}^{(4)}(u, t, \tilde{u}, \tilde{t})
$$

where $\mathcal{K}$ is the reduced transcendental kernel of the double box (see below (4.12)) and $\mathcal{W}_{p p p p}^{(4)}(s, t, \tilde{s}, \tilde{t})$ is a rational function with the symmetry

$$
\mathcal{W}_{p p p p}^{(4)}(s, t, \tilde{s}, \tilde{t})=\mathcal{W}_{p p p p}^{(4)}(t, s, \tilde{t}, \tilde{s}) .
$$

The reduced kernel $\mathcal{K}$ of the double box $[51,52]$ is

$$
\mathcal{K}[z, w]=\psi^{(1)}(-z)+\psi^{(1)}(-w)-\left(\psi^{(0)}(-z)+\psi^{(0)}(-w)\right)^{2}-\pi^{2} .
$$

This function has been studied in [53], and upon inspection only contains simple poles in $s$ and $t$. The Mellin transform of the double box is indeed just given by the product of $\Gamma[-s]^{2} \Gamma[-t]^{2} \Gamma[s+t+1]^{2}$ and the reduced kernel $\mathcal{K}[z, w] .{ }^{14}$

In position space, the top weight four part of the one-loop correlators are given by the double box in the three independent orientations, each orientation multiplied by a rational functions of the cross ratios, fixed by the double logarithmic discontinuity. The structure of the top-weight 4 integrand in (4.10) perfectly reflects the position space understanding of the correlator. In particular, $\mathcal{W}_{p}^{(4)}(s, t, \tilde{s}, \tilde{t})$ is determined by the knowledge of $\mathcal{T}^{(4)}(\mathbf{s}, \mathbf{t})$ in (4.5) and (4.8), and the action of $\Delta^{(8)}$ as described in the previous section.

We match the $\log ^{2} U \log ^{2} V$ projection of the correlator with that of the double logarithmic discontinuity. This is equivalent to looking at the residue of the triple poles in $s$ and $t$ in (4.9), and triple poles in $t$ of the double logarithmic discontinuity given in (4.8). From the explicit Mellin integrals we can present the final result in a simple fashion as a sum over (twenty-five) shifts,

$$
\mathcal{W}_{p p p p}^{w=4}(s, t, \tilde{s}, \tilde{t})=\sum_{\substack{-4 \leq a \leq 0 \\-3-a \leq b \leq 3}} \delta_{p p p p}(a, b) \mathcal{T}^{(4)}[\mathbf{s}+a, \mathbf{t}+b]
$$

where the coefficients $\delta_{\text {pppp }}(a, b)$ depend on $p$ and the Mellin variables, and are attached in the supplementary material. For finite $p$ the rational function $\mathcal{W}_{p p p p}^{w=4}(s, t, \tilde{s}, \tilde{t})$ is a four variable function, because of the $\delta_{\text {pppp }}(a, b)$.

Coming to the large $p$ limit, we have to consider the limit of $\mathcal{W}_{p p p p}^{w=4}(s, t, \tilde{s}, \tilde{t})$ when all the variables are large and scale linearly with $p$. This limit is obviously factorised into that of $\mathcal{K}$ and $\mathcal{W}_{\text {pppp }}^{(4)}$, and the split of the full kernel into $\Gamma_{\otimes}$ and the reduced kernel is crucial for this analysis.

The reduced kernel $\mathcal{K}$ in the large $p$ limit is dominated by the asymptotic of $\psi^{(0)}$, which is leading compared to the asymptotic of $\psi^{(1)}$,

$$
\lim _{p \rightarrow \infty} \mathcal{K}[p z, p w]=-(\log (-p z)-\log (-p w))^{2}-\pi^{2}=-\log ^{2}((-z) /(-w))-\pi^{2} .
$$

This combination is 'dimensionless' and thus does not have additional $p$-dependence.

\footnotetext{
${ }^{14} \mathcal{K}[z, w]$ can be bootstrapped from the small $U$ and $V$ expansion of the ladder integrals, since these are pure transcendentals over $\left(x_{1}-x_{2}\right)$. It is simple to see that $\psi^{(0)}(-z) \psi^{(0)}(-w)$ is not enough, but can only be corrected by a linear combinations of weight 2 terms, which at most bring in simple poles, i.e. $\psi^{(0)}(-z) \psi^{(0)}(-z)-\psi^{(1)}(-z)$, the analogous for $w$, and $\pi^{2}$.
} 
The limit on $\mathcal{W}_{\text {pppp }}^{(4)}$ has more features. Without loss of generality, we focus on one of the three orientation. The result can be put in the following suggestive form,

$$
\lim _{p \rightarrow \infty} \mathcal{W}_{p p p p}^{(4)}\left(s_{\mathrm{cl}}, t_{\mathrm{cl}}, \tilde{s}_{\mathrm{cl}}, \tilde{t}_{\mathrm{cl}}\right)=\left(\sum_{\substack{4 \leq a \leq 0 \\-3-a \leq b \leq 3}} \lim _{\substack{1 \rightarrow \infty \\ \text { s. }}} \delta_{p p p p}(a, b)\right)\left(\lim _{p \rightarrow \infty} \mathcal{T}^{(4)}\left(\mathbf{s}_{\mathrm{cl}}, \mathbf{t}_{\mathrm{cl}}\right)\right) .
$$

The expression (4.15) is correct because as we now show both the sum over $\delta_{p p p p}(a, b)$ and $\mathcal{T}^{(4)}(\mathbf{s}, \mathbf{t})$ have a non vanishing leading contribution. If we were to be taking the large $s$ and $t$ limit, rather than the large $p$ limit, we would not be finding such a simple relation.

The large $p$ limit gives

$$
\begin{aligned}
\lim _{p \rightarrow \infty} \mathcal{W}_{p p p p}^{(4)}\left(s_{\mathrm{cl}}, t_{\mathrm{cl}}, \tilde{s}_{\mathrm{cl}}, \tilde{t}_{\mathrm{cl}}\right) & =+\frac{1}{120} \times\left(16 p^{4}\left(s_{\mathrm{cl}}+\tilde{s}_{\mathrm{cl}}\right)^{4}\right) \times \frac{\left(\mathbf{t}_{\mathrm{cl}}\right)^{2}}{\left(\mathbf{s}_{\mathrm{cl}}\right)^{2}\left(-\mathbf{u}_{\mathrm{cl}}\right)^{3}} \\
& =-\frac{1}{120} \times \frac{1}{\mathbf{s}_{\mathrm{cl}} \mathbf{t}_{\mathrm{cl}} \mathbf{u}_{\mathrm{cl}}} \times\left(\Sigma^{4} \mathbf{s}_{\mathrm{cl}}^{4}\right) \times \frac{\left(\mathbf{t}_{\mathrm{cl}}\right)^{3}}{\left(\mathbf{s}_{\mathrm{cl}}\right)\left(\mathbf{u}_{\mathrm{cl}}\right)^{2}}
\end{aligned}
$$

It can be immediately seen here that the contribution from $\delta(a, b)$ scales like $p^{8}$, as it is natural to expect from $\Delta^{(8)}$ when all variables are scaled together. But the nicest aspect of this calculation is the following.

The sum over the shifts $a$ and $b$ in (4.15) reorganises to give a simple multiplicative $(2 p)^{4}\left(\mathbf{s}_{\mathrm{cl}}\right)^{4}$ factor. This is very remarkable because all terms in the sum are actually non trivial! When we include $p_{21} \neq 0$ and $p_{34} \neq 0$, as described in details in appendix $\mathrm{D}$, the result is indeed $\Sigma^{4} \mathbf{s}_{\mathrm{cl}}^{4}$, as written in (4.16). In this case the combinatorics that leads to such a simple result is even more surprising, and in fact let us point out that $\Sigma$ is not explicit in any of the terms involved in the sum. Individually, these depend on $c_{s}, c_{t}, c_{u}$ and $p_{3}$.

The action of $\Delta^{(8)}$ restores the extra symmetry of the $\log ^{2} U \log ^{2} V$ coefficient function, which we highlighted in (4.11), that is lost at the level of the preamplitude $\mathcal{T}^{(4)}$. We see in (4.16) that at leading order in the large $p$ expansion this symmetry becomes a symmetry of the ten-dimensional variables, $\mathbf{s}_{\mathrm{cl}} \leftrightarrow \mathbf{t}_{\mathrm{cl}}$.

\subsubsection{Top-weight 3}

The structure of the top-weight 3 amplitude is simpler. The reduced kernel is simply a polygamma function, and the full $\mathcal{W}_{p p p p}^{w=3}$ is

$$
\mathcal{W}_{p p p p}^{w=3}=\psi(-\mathbf{s}) \mathcal{W}_{p p p p}^{(3)}(s, t, \tilde{s}, \tilde{t})+\psi(-\mathbf{t}) \mathcal{W}_{p p p p}^{(3)}(t, u, \tilde{t}, \tilde{u})+\psi(-\mathbf{u}) \mathcal{W}_{p p p p}^{(3)}(u, t, \tilde{u}, \tilde{t}),
$$

where the rational function $\mathcal{W}_{p p p p}^{(3)}(s, t, \tilde{s}, \tilde{t})$ has the symmetry

$$
\mathcal{W}_{\text {pppp }}^{(3)}(s, t, \tilde{s}, \tilde{t})=\mathcal{W}_{\text {pppp }}^{(3)}(s, u, \tilde{s}, \tilde{u}) .
$$

In position space, transcendental functions with weight $w=4^{-}, 3^{ \pm}, 2^{+}$have coefficient functions fully determined by the double discontinuity in the various orientations. In the same way, $\mathcal{W}_{p p p p}^{w=3}$ is determined by $\mathcal{T}^{(4)}$ and $\mathcal{T}^{(3)}$, since $\mathcal{W}_{\text {pppp }}^{w=3}$ first contributes to the $\log ^{2} U \log ^{1} V$ projection of the correlator, which is again fixed by the double discontinuity. 
To see this consider the residue of triple poles in $s$ and double poles in $t$ of the amplitude (4.9), and compare with the residue of the double poles in $t$ of the double logarithmic discontinuity (4.8).

The matching procedure just described follows the same logic as for $\mathcal{W}_{p p p p}^{w=4}$ but for a minor modification: shifting the contour in order to absorb $\Delta^{(8)}(p)$ produces a shift of the arguments of the polygammas in (4.8). These shifts do not affect the determination of $\mathcal{W}_{p p p p}^{w=4}$ itself, given in the previous section, but now they must be included correctly. Taking care of this subtlety we find

$$
\begin{aligned}
& \mathcal{W}_{p p p p}^{(3)}(s, t, \tilde{s}, \tilde{t})=\sum_{\substack{-4 \leq a \leq 0 \\
-3-a \leq b \leq 3}} \delta_{\text {pppp }}(a, b)\left(2 \mathcal{T}^{(3)}[\mathbf{s}+a, \mathbf{t}+b]+\right. \\
& \left.r_{p p p p}(a,-b, \mathbf{s},-\mathbf{t}) \mathcal{T}^{(4)}[\mathbf{s}+a, \mathbf{t}+b]+r_{p p p p}(a, a+b, \mathbf{s},-\mathbf{u}) \mathcal{T}^{(4)}[\mathbf{s}+a, \mathbf{u}-a-b]\right)
\end{aligned}
$$

where $r_{\text {pppp }}(\alpha, \beta, \mathbf{s}, \mathbf{t})$ is the rational function due to the shifts in the arguments of the polygammas. ${ }^{15}$

Coming to the large $p$ limit, we focus on one of the three orientations without loss of generality. The reduced transcendental kernel is just a polygamma $\psi^{(0)}$ which asymptotes to a logarithm, as before. With a same argument as in (4.15), the limit of the rational function can be put in the suggestive form,

$$
\lim _{p \rightarrow \infty} \mathcal{W}_{p p p p}^{w=3}\left(s_{\mathrm{cl}}, t_{\mathrm{cl}}, \tilde{s}_{\mathrm{cl}}, \tilde{t}_{\mathrm{cl}}\right)=\left(\sum_{\substack{-4 \leq a \leq 0 \\-3-a \leq b \leq 3}} \lim _{p \rightarrow \infty} \delta_{p p p p}(a, b)\right)\left(\lim _{p \rightarrow \infty} 2 \mathcal{T}^{(3)}\left[\mathbf{s}_{\mathrm{cl}}, \mathbf{t}_{\mathrm{cl}}\right]\right) .
$$

Notice also that contributions to $\mathcal{W}_{\text {pppp }}^{(3)}$ coming from $\mathcal{T}^{(4)}$ are subleading compared to $\mathcal{T}^{(3)}$, since they come with one extra power of $p$ in the denominator, due to $r_{p p p p}$. More explicitly, we obtain the result

$$
\lim _{p \rightarrow \infty} \mathcal{W}_{p p p p}^{(3)}\left(s_{\mathrm{cl}}, t_{\mathrm{cl}}, \tilde{s}_{\mathrm{cl}}, \tilde{t}_{\mathrm{cl}}\right)=\frac{1}{120} \times \frac{\Sigma^{4}}{\mathbf{s}_{\mathrm{cl}} \mathbf{t}_{\mathrm{cl}} \mathbf{u}_{\mathrm{cl}}} \times \frac{7\left(\mathbf{t}_{\mathrm{cl}}\right)^{2}\left(\mathbf{s}_{\mathrm{cl}}\right)^{4}+7\left(\mathbf{u}_{\mathrm{cl}}\right)^{2}\left(\mathbf{s}_{\mathrm{cl}}\right)^{4}-3\left(\mathbf{s}_{\mathrm{cl}}\right)^{6}}{2\left(\mathbf{t}_{\mathrm{cl}}\right)\left(\mathbf{u}_{\mathrm{cl}}\right)} .
$$

The r.h.s. shows the symmetry (4.17) at the level of $10 \mathrm{~d}$ variables, $\mathbf{t}_{\mathrm{cl}} \leftrightarrow \mathbf{u}_{\mathrm{cl}}$.

\subsubsection{Top-weight 2}

The top-weight 2 amplitude is just a rational function, since the full kernel in this case coincides with $\Gamma_{\otimes}$, i.e. $\mathcal{W}_{p p p p}^{w=2}=\mathcal{W}_{p p p p}^{(2)}$. For illustration we quote the result for $p=2$,

$$
\mathcal{W}_{2222}^{(2)}(s, t)=\frac{\operatorname{num}\left(I_{i}\right)}{(s+1)(s+4)(s+5)(t+1)(t+4)(t+5)(u+1)(u+4)(u+5)}+\beta^{(1)}
$$

\footnotetext{
${ }^{15}$ This is just

$$
r_{p p p p}(\alpha, \beta, w, z)=-\sum_{n=0}^{|\alpha|-1} \frac{1}{\operatorname{sgn}[\alpha]\left(w+\frac{\operatorname{sgn}[\alpha]+1}{2}\right)+n}+\sum_{m=0}^{|\beta|-1} \frac{1}{\operatorname{sgn}[\beta]\left(z+\frac{\operatorname{sgn}[\beta]-1}{2}\right)+m} .
$$
}


where $I_{i}=s^{i}+t^{i}+u^{i}$ with $u=-s-t-4$ for this correlator, and

$$
\begin{aligned}
\text { num }= & +\frac{3}{25} I_{5} I_{5}-\frac{68849782843}{3072000} I_{4} I_{5}-\frac{524233}{2250} I_{3} I_{6}+\frac{16495714123}{1024000} I_{2} I_{7} \\
& -\frac{63132297731}{768000} I_{4} I_{4}-\frac{6745323479}{72000} I_{3} I_{5}+\frac{63132758531}{768000} I_{2} I_{6} \\
& -\frac{558138949661}{1152000} I_{3} I_{4}-\frac{879855811}{38400} I_{2} I_{5} \\
& -\frac{11005992151}{18000} I_{3} I_{3}-\frac{191551927561}{288000} I_{2} I_{4} \\
& -\frac{21075629959}{14400} I_{2} I_{3}-\frac{11046578393}{18000} I_{2} I_{2} .
\end{aligned}
$$

This is not a unique decomposition, however it makes crossing invariance of $\mathcal{W}_{2222}^{(2)}$ manifest. The (only) constant ambiguity $\beta$ can be fixed by using results for the integrated correlator, which are provided independently by localisation [45].

What happens for $\mathcal{W}_{p_{1} p_{2} p_{3} p_{4}}^{(2)}$, for fixed values of the external charge, is quite non trivial and deserves a separate section which we summarize here below.

The position space algorithm of [16], after imposing crossing and absence of (euclidean) unphysical $x_{1}=x_{2}$ poles, returns a correlator with free parameters in the coefficient functions relative to transcendental weights $w=2^{-}, 1^{+}, 0$. These free coefficients provide the same degrees of freedom of $\mathcal{W}_{p_{1} p_{2} p_{3} p_{4}}^{(2)}$. In general, fixing the full correlator (up to stringy-type ambiguities) requires

1) Knowledge about the precise definition of the external single particle operators,

2) One-loop OPE predictions in- and below-window for the long sector,

3) One-loop OPE predictions for protected semi-short multiplets at the unitarity bound, in order to perform correctly multiplet recombination.

There is a non trivial exchange of information in items 2) and 3) between free theory and the dynamical amplitude, versus the spectrum of supergravity. These considerations motivated [16] to introduce the minimal one-loop function, which is nicely understood to descend from the double discontinuity, upon modifying the tree level Mellin amplitude by an upgraded tree level function. This upgraded tree level function takes care of some trivial multiplet recombination, for example the multiplet recombination to cancel stringy states in supergravity, and ascribe all the new features of the one-loop dynamics to the minimal one-loop function. The upgraded tree level function extends consistently $\mathcal{M}_{p_{1} p_{2} p_{3} p_{4}}^{(1)}$ to a non-planar function, and it is an exact function of $N$. Re-expanding the latter at one-loop gives a contribution to $\mathcal{W}_{p_{1} p_{2} p_{3} p_{4}}^{(2)}$.

One-loop stringy-type ambiguities contribute only to finite spin and make their appearance only in $\mathcal{W}_{p_{1} p_{2} p_{3} p_{4}}^{(2)}$. Generically they are described by an ansatz of the form

$$
\left.\mathcal{W}_{p_{1} p_{2} p_{3} p_{4}}^{(2)}(s, t, \tilde{U}, \tilde{V})\right|_{\text {ambiguities }}=\sum_{T}\left(\beta_{\tilde{s}, \tilde{t} ; \vec{p}}^{(1)}+\beta_{\tilde{s}, \tilde{t} ; \vec{p}}^{(s)} s+\beta_{\tilde{s}, \tilde{t} ; \vec{p}}^{(t)} t\right) \tilde{U}^{\tilde{s}} \tilde{V}^{\tilde{t}}
$$


where $\beta^{\text {type }}$ are arbitrary constants. As far as the counting goes we can think of terms labelled by $\beta^{(1)}$ as corresponding to $R^{4}$ in the bulk $10 \mathrm{~d}$ effective action, and terms labelled by $\beta^{(s)}$ and $\beta^{(t)}$ as corresponding to $\partial^{2} R^{4}$. If a correlator is symmetric under a crossing transformation the same symmetry needs to be imposed on (4.23). For example, equal charges correlators are fully crossing invariant.

As detailed already in [16], the position space bootstrap for the minimal one-loop function, after taking into account items 1), 2) and 3), returns a function with finitely many free parameters, which case by case can be shown to be in correspondence with the parameters $\beta$ in (4.23). We do not know a closed and explicit expression for $\mathcal{W}_{\text {pppp }}^{(2)}$, yet. However, we will now argue that we can deduce the large $p$ limit of $\mathcal{W}_{\text {pppp }}^{(2)}$ by covariantising the large $s$ and $t$ limit at fixed $p$. We already seen this idea at work in the case of the VS amplitude, here the procedure is slightly more involved since we are dealing with rational functions rather than polynomials.

After computing $\mathcal{W}_{p p p p}^{(2)}$ for $p=2,3,4$, we notice that the corresponding large $s$ and $t$ limit is controlled by a very simple function, ${ }^{16}$

$$
\begin{aligned}
\lim _{s, t \rightarrow \infty} \mathcal{W}_{2222}^{(2)}(s, t)= & \frac{3\left(s^{2}+s t+t^{2}\right)^{2}}{s t(s+t)} \\
\lim _{s, t \rightarrow \infty} \mathcal{W}_{3333}^{(2)}(s, t, \tilde{U}, \tilde{V})= & \frac{14\left(s^{2}+s t+t^{2}\right)^{2}}{s t(s+t)}(1+\tilde{U}+\tilde{V})+\beta_{3333}^{(s)}(\tilde{U} s+\tilde{V} t-(s+t)) \\
\lim _{s, t \rightarrow \infty} \mathcal{W}_{4444}^{(2)}(s, t, \tilde{U}, \tilde{V})= & \frac{42\left(s^{2}+s t+t^{2}\right)^{2}}{s t(s+t)}\left(1+\tilde{U}^{2}+\tilde{V}^{2}+4 \tilde{U}+4 \tilde{V}+4 \tilde{U} \tilde{V}\right) \\
& +\beta_{4444}^{(s)}\left(\tilde{U}^{2} s+\tilde{V}^{2} t-(s+t)\right)+\beta_{4444}^{(t)}(\tilde{U} \tilde{V}(s+t)-\tilde{U} t-\tilde{V} s) .
\end{aligned}
$$

Importantly, the large $s$ and $t$ limit scale linearly with $s$, and thus depends only on non constant stringy ambiguities. In particular, it does not see the presence of the upgraded tree level function, which at most scales like $1 / s^{2}[16]$.

A pattern similar to that in $(4.24)-(4.27)$ shows up in $\mathcal{W}_{2222,3333,4444}^{(4)}$, and $\mathcal{W}_{2222,3333,44444}^{(3)}$ if we repeat the large $s$ and $t$ analysis at fixed external charges. Indeed, the following general result holds ${ }^{17}$

$$
\begin{aligned}
\lim _{s, t \rightarrow \infty} \mathcal{W}_{p p p p}^{w=4,3}(s, t, \tilde{s}, \tilde{t}) & =\frac{p(p+1)(2 p-1)(2 p+1)}{30} f^{w=4,3}(s, t) \sum_{T} \frac{\tilde{U}^{\tilde{s}} \tilde{V}^{\tilde{t}}}{\tilde{s} !^{2} \tilde{t} !^{2} \tilde{u} !^{2}} \\
f^{(4)} & =\frac{s^{2} t^{2}}{(s+t)^{3}} \\
f^{(3)} & =\frac{7 t^{2} s^{3}+7(s+t)^{2} s^{3}-3 s^{5}}{2 t^{2}(s+t)^{2}}
\end{aligned}
$$

\footnotetext{
${ }^{16}$ We have used the results in [16], cfr. section 4.4 and 4.5. The limit here compares with the limit taken on the minimal one-loop functions attached in [16] by a redefinition of the ambiguities, which is always possible.

${ }^{17}$ This prefactor can be written as the prefactor coming from the flat space limit a la Penedones:
}

$$
\frac{p(p+1)(2 p-1)(2 p+1)}{30}=\left.\frac{(\Sigma-1)_{4}}{120}\right|_{p_{i=1,2,3,4}=p} \quad ; \quad \Sigma=\frac{p_{1}+p_{2}+p_{3}+p_{4}}{2} .
$$


This same pattern goes over the top-weight 2 function and we can match (4.24)-(4.27) with

$$
\begin{aligned}
\lim _{s, t \rightarrow \infty} \mathcal{W}_{p p p p}^{(2)}(s, t, i, j) & =\frac{p(p+1)(2 p-1)(2 p+1)}{30} f^{(2)}(s, t) \sum_{T} \frac{\tilde{U}}{\tilde{s} !^{2} \tilde{t} \tilde{V}^{2} \tilde{u} !^{2}} \\
f^{(2)} & =\frac{\left(s^{2}+s t+t^{2}\right)^{2}}{s t(s+t)} .
\end{aligned}
$$

For $\mathcal{W}_{p p p p}^{(4)}$, and $\mathcal{W}_{p p p p}^{(3)}$ we know how to compare the large $s$ and $t$ limit with the large $p$ limit. Covariantising we find the large $p$ limit of $\mathcal{W}_{p p p p}^{(2)}$ to be

$$
\lim _{p \rightarrow \infty} \mathcal{W}_{p p p p}^{(2)}\left(s_{\mathrm{cl}}, t_{\mathrm{cl}}, \tilde{s}_{\mathrm{cl}}, \tilde{t}_{\mathrm{cl}}\right)=-\frac{1}{120} \times \frac{\Sigma^{4}}{\mathbf{s}_{\mathrm{cl}} \mathbf{t}_{\mathrm{cl}} \mathbf{u}_{\mathrm{cl}}} \times \frac{\left(\left(\mathbf{s}_{\mathrm{cl}}\right)^{2}+\left(\mathbf{u}_{\mathrm{cl}}\right)^{2}+\left(\mathbf{t}_{\mathrm{cl}}\right)^{2}\right)^{2}}{4} .
$$

In this formula we dropped the ambiguities, which instead we saw in (4.24)-(4.27) contributing to the flat space limit a la Penedones. Notice now that in the position space bootstrap there is an abundance of such ambiguities since they are constrained only from crossing and spin truncation. This is essentially how the counting in (4.23) works. However, all these ambiguities should be consistent with the four-fold representation, something that at fixed $p$ cannot be appreciated. From this point of view, we can refine our previous counting and consider only crossing invariants $A d S_{5} \times S^{5}$ ambiguities, for example

$$
\left.\mathcal{W}_{p p p p}^{(2)}(s, t, \tilde{s}, \tilde{t})\right|_{\text {ambiguities }}=\beta_{p p p p}^{R^{4}}+\beta_{p p p p}^{\partial^{2} R^{4}}\left(\mathbf{s}\left(\frac{1}{2} c_{s}+\tilde{s}\right)+\mathbf{t}\left(\frac{1}{2} c_{t}+\tilde{t}\right)+\mathbf{u}\left(\frac{1}{2} c_{u}+\tilde{u}\right)\right) .
$$

The stringy origin of these ambiguities is the $R^{4}$ term and its derivatives compactified on $A d S_{5} \times S^{5}$. Notice in fact the similarity with (3.11)-(3.12) in the context of the VS amplitude. It is tempting to set $\beta^{\partial^{2} R^{4}}$ to zero but the only argument that can be made is to use independent results from localisation to fix it, as in the case of $\beta^{R^{4}}$ [45].

In the next section we collect all the various contributions to $\mathcal{M}_{p p p p}^{(2)}$ in the large $p$ limit and we discuss the relation with the ten-dimensional box integral in flat space.

\subsection{Gravitational S-matrix in the large $p$ limit}

Let us recall the saddle point relation (1.11) between position and Mellin space representation of the amplitude, in the large $p$ limit,

$$
\lim _{p \rightarrow \infty} \mathcal{A}(U, V, \tilde{U}, \tilde{V})=\frac{4 \pi^{4}}{(U V \tilde{U} \tilde{V})^{\frac{1}{4}}} \frac{(1+\sqrt{\tilde{U}}+\sqrt{\tilde{V}})^{2(p-2)+\frac{3}{2}}}{(1+\sqrt{U}+\sqrt{V})^{2(p+2)-\frac{3}{2}}} \times p^{4} \lim _{p \rightarrow \infty} \mathcal{M}\left(s_{\mathrm{cl}}, t_{\mathrm{cl}}, \tilde{s}_{\mathrm{cl}}, \tilde{t}_{\mathrm{cl}}\right) .
$$

We will now show how the r.h.s. looks like at one loop.

The result for $\mathcal{M}^{(2)}$, obtained in the previous section, once organised by weight $w$, and divided out by the tree level amplitude becomes

$$
\lim _{p \rightarrow \infty} \mathcal{M}_{p p p p}^{(2)}\left(s_{\mathrm{cl}}, t_{\mathrm{cl}}, \tilde{s}_{\mathrm{cl}}, \tilde{t}_{\mathrm{cl}}\right)=\frac{1}{\mathbf{s}_{\mathrm{cl}} \mathbf{t}_{\mathrm{cl}} \mathbf{u}_{\mathrm{cl}}} \times \Phi\left(\mathbf{s}_{\mathrm{cl}}, \mathbf{t}_{\mathrm{cl}}\right)
$$


where

$$
\begin{aligned}
\Phi\left(\mathbf{s}_{\mathrm{cl}}, \mathbf{t}_{\mathrm{cl}}\right)=\frac{\Sigma^{4}}{5 !}[ & +\frac{\left(\mathbf{s}_{\mathrm{cl}}\right)^{3}\left(\mathbf{t}_{\mathrm{cl}}\right)^{3}}{\left(\mathbf{u}_{\mathrm{cl}}\right)^{2}}\left(\pi^{2}+\log ^{2}\left(\left(-\mathbf{s}_{\mathrm{cl}}\right) /\left(-\mathbf{t}_{\mathrm{cl}}\right)\right)\right)+\operatorname{crossing}+ \\
& +\frac{7\left(\mathbf{t}_{\mathrm{cl}}\right)^{2}\left(\mathbf{s}_{\mathrm{cl}}\right)^{4}+7\left(\mathbf{u}_{\mathrm{cl}}\right)^{2}\left(\mathbf{s}_{\mathrm{cl}}\right)^{4}-3\left(\mathbf{s}_{\mathrm{cl}}\right)^{6}}{2\left(\mathbf{t}_{\mathrm{cl}}\right)\left(\mathbf{u}_{\mathrm{cl}}\right)} \log \left(-\mathbf{s}_{\mathrm{cl}}\right)+\operatorname{crossing}+ \\
& \left.-\frac{\left(\left(\mathbf{s}_{\mathrm{cl}}\right)^{2}+\left(\mathbf{t}_{\mathrm{cl}}\right)^{2}+\left(\mathbf{u}_{\mathrm{cl}}\right)^{2}\right)^{2}}{4}+\operatorname{crossing}\right]
\end{aligned}
$$

We want to compare (4.36)-(4.37) with the flat space amplitude of type IIB SUGRA in $10 \mathrm{~d}$, from string theory $[54,55]$. The idea of this computation was pioneered in [19], and here we will consider its Mellin space version, uplifted to 10d. Assembling the various results we find ${ }^{18}$

$$
\begin{aligned}
\mathcal{S}^{\text {flat }}(s, t) & =1+\mathcal{S}^{\text {1-loop,flat }}(s, t)+\ldots \\
\mathcal{S}^{1 \text {-loop,flat }}(s, t) & =8 \pi g_{s}^{2}\left(\mathbb{B}\left(\alpha^{\prime} s, \alpha^{\prime} t\right)+\mathbb{B}\left(\alpha^{\prime} s, \alpha^{\prime} u\right)+\mathbb{B}\left(\alpha^{\prime} t, \alpha^{\prime} u\right)\right)
\end{aligned}
$$

where $\mathbb{B}$ is related to the box function in $10 \mathrm{~d}$. In particular,

$$
\begin{aligned}
\mathbb{B}(s, t)=\frac{\pi}{5 ! 2^{1+6}}[ & {\left[\frac{s^{3} t^{3}}{u^{2}}\left(\pi^{2}+\log ^{2} \frac{-s}{-t}\right)+\right.} \\
& \left.\frac{s^{3} t}{u}(u-2 t) \log (-s)+\frac{t^{3} s}{u}(u-2 s) \log (-t)-s^{2} t^{2}+C_{2} s t u^{2}\right] .
\end{aligned}
$$

The term denoted with $C_{2}=1$ cancels in the sum over orientations, when $s+t+u=0 .{ }^{19}$

By looking at $\mathcal{S}^{1 \text {-loop,flat }}(s, t)$ and comparing with $\lim _{p \rightarrow \infty} \mathcal{M}_{p p p p}^{(2)}\left(\mathbf{s}_{\mathrm{cl}}, \mathbf{t}_{\mathrm{cl}}\right)$ we find the relation

$$
\lim _{p \rightarrow \infty}\left(\frac{1}{N^{2}} \mathcal{M}_{p p p p}^{(2)}\left(s_{\mathrm{cl}}, t_{\mathrm{cl}}, \tilde{s}_{\mathrm{cl}}, \tilde{t}_{\mathrm{cl}}\right)\right)=\frac{1}{\mathbf{s}_{\mathrm{cl}} \mathbf{t}_{\mathrm{cl}} \mathbf{u}_{\mathrm{cl}}} \mathcal{S}^{1-\text { loop }, \text { flat }}\left(\frac{4 \Sigma}{R^{2}} \mathbf{s}_{\mathrm{cl}}, \frac{4 \Sigma}{R^{2}} \mathbf{t}_{\mathrm{cl}} ; g_{s}\right)
$$

with $R^{4} / \alpha^{2}=4 \pi g_{s} N$. This is the same identification of $10 \mathrm{~d}$ Mandelstam variables we found in the VS amplitude, and again agrees with our expectations from the picture of four propagating geodesics towards a common bulk point. Thus we have showed the coincidence between the large $p$ limit of the $A d S_{5} \times S^{5}$ amplitude and the 10d flat space scattering amplitude, at one-loop.

\footnotetext{
${ }^{18}$ On the stringy side, we refer to (4.1), (4.22) and (4.25) of [56]. In their (4.22) we seem to find a $8 \pi$ instead of $2 \pi$. This would match formula (5.28) of [19] (with an $s^{4} I_{\mathrm{box}}$ ). In formula (4.25) of [56], we used the identity $\operatorname{Li}_{2}(z)+\operatorname{Li}_{2}(1 / z)=-\frac{1}{2} \log ^{2}(-z)-\frac{\pi^{2}}{6}$. Notice also that upon shifting $\alpha^{\prime} \rightarrow \alpha^{\prime} / \Lambda^{2}$ we would find that $s+t+u=0$ implies

$$
\log \Lambda^{2}\left(\frac{s^{3} t}{u}(u-2 t)+\frac{t^{3} s}{u}(u-2 s)+\text { crossing }\right)=0 .
$$

Decomposing $\log (-s)=\frac{1}{2}(\log (-s)-\log (-t))+\frac{1}{2}(\log (-s)+\log (-t))$, and similaly for $\log (-t)$, it is clear that the property above is a property of the coefficient function for the symmetric term.

${ }^{19}$ The 10d box presented in [19] includes also the quadratic divergence, restored after dim-reg.
} 
The scaling with $p$ of the Mellin amplitude can be generalised to the $\ell$-loop order, and leads to the following scheme:

$$
\begin{aligned}
& \lim _{p \rightarrow \infty} \mathcal{M}_{\vec{p}}^{\ell-\text { loop }}\left(s_{\mathrm{cl}}, t_{\mathrm{cl}}, \tilde{s}_{\mathrm{cl}}, \tilde{t}_{\mathrm{cl}}\right)=\frac{1}{\mathbf{s}_{\mathrm{cl}} \mathbf{t}_{\mathrm{cl}} \mathbf{u}_{\mathrm{cl}}} \times\left(\frac{\Sigma^{4} \mathbf{s}_{\mathrm{cl}}^{4}}{N^{2}}\right)^{\ell} \\
& {\left[\sum_{0 \leq d \leq 2 \ell} \text { lim-Transcendetal } \mathbf{l}_{\ell, d}\left(\mathbf{s}_{\mathrm{cl}}, \mathbf{t}_{\mathrm{cl}}\right) \times \operatorname{Rational}_{\ell, d}\left(\frac{\mathbf{t}_{\mathrm{cl}}}{\mathbf{s}_{\mathrm{cl}}}\right)+\text { crossing }\right] }
\end{aligned}
$$

where lim-Transcendetal are the trascendental functions appearing in the flat space scattering amplitude, which come from the limit of the Transcendetal functions in the $A d S_{5} \times S^{5}$ Mellin amplitude, when the Mellin variables are large. The latter are meromorphic, with poles dictated by the OPE, while the first ones generically have also branch cuts, related to the physics of the 10d scattering amplitude. From the $A d S_{5} \times S^{5}$ point of view these branch cuts arise from the accumulation of the poles when the Mellin variables are large. The transcendental weight goes down by two in the process, because of $\Gamma_{\otimes}$ which plays a different role, compared to the reduced transcendental kernels. It is quite interesting to notice that, differently from perturbation theory where the transcendental content of the amplitude has fixed weight at given loop order, at strong coupling all lower weights are turned on, and there is a correspondence between transcendental functions across dimensions. The rational functions denoted schematically by Rational in (4.41), have degree zero under the large $p$ limit. For example at one-loop we have found

$$
\lim _{p \rightarrow \infty} \mathcal{W}_{p p p p}^{(4)}\left(s_{\mathrm{cl}}, t_{\mathrm{cl}}, \tilde{s}_{\mathrm{cl}}, \tilde{t}_{\mathrm{cl}}\right) \sim \frac{1}{\mathbf{s}_{\mathrm{cl}} \mathbf{t}_{\mathrm{cl}} \mathbf{u}_{\mathrm{cl}}} \times p^{4} \mathbf{s}_{\mathrm{cl}}^{4} \times\left[\operatorname{Rational}_{1,4}=\frac{\left(\mathbf{t}_{\mathrm{cl}}\right)^{3}}{\left(\mathbf{s}_{\mathrm{cl}}\right)\left(\mathbf{u}_{\mathrm{cl}}\right)^{2}}\right]
$$

and similarly for $\mathcal{W}_{\text {pppp }}^{(3)}$ and $\mathcal{W}_{\text {pppp. }}^{(2)}{ }^{20}$

Finally, note that since both $\Sigma$ and the bold font variables scale with $p$ we see that the genus $\ell$ contribution comes dressed by an effective $\left(p^{8} / N^{2}\right)^{\ell}$ coupling, from which we estimate that perturbative supergravity is a valid approximation to the correlator as long as $p \sim N^{1 / 4}$. For us, since $N$ is the largest parameter, this is always small. In some recent explorations discussed in the next section, $p \sim N^{1 / 2}$; this effective coupling would be large and we would need to fully resum the non-planar series.

\section{Discussion}

In this paper we studied the large $p$ limit of the four-point correlators $\left\langle\mathcal{O}_{p_{1}} \mathcal{O}_{p_{2}} \mathcal{O}_{p_{3}} \mathcal{O}_{p_{4}}\right\rangle$ of single-particle operators in $\mathcal{N}=4 \mathrm{SYM}$ in the large $N$ limit at strong 't Hooft coupling. We considered different regimes of $p$ having in mind the greater picture illustrated by figure 1 . The idea is to interpolate from the top left corner (AdS SUGRA) to the bottom right corner (AdS minimal areas), and see how the interpolation goes. In this paper we took a first step and we focused mostly on perturbative string theory, pushing SUGRA results up to the flat space Gross-Mende phase, where the nature of string theory as an extended object appears for the first time, but does not yet backreact on the $A d S_{5} \times S^{5}$ geometry.

\footnotetext{
${ }^{20}$ In appendix D.1 we provide a test of our scheme for the case of the leading logs at two-loop.
} 

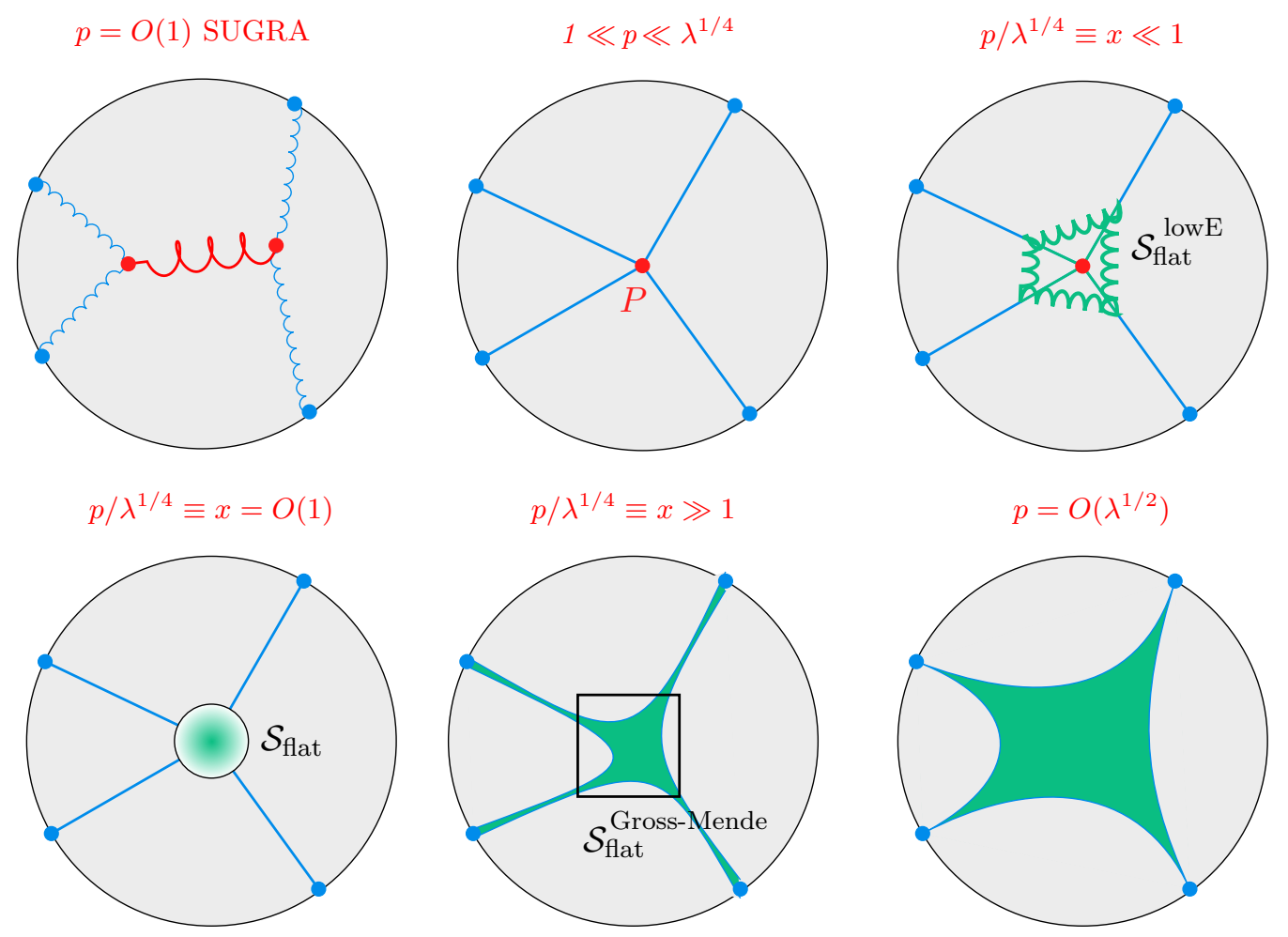

Figure 1. From AdS SUGRA $(p=O(1))$ to AdS string minimal areas $p=O(\sqrt{\lambda})$. We go from SUGRA taking first $p$ large, then we dress the amplitude by low energy flat space string theory $\left(p / \lambda^{1 / 4} \ll 1\right)$, full flat space string theory $\left(p / \lambda^{1 / 4} \sim 1\right)$ and high energy flat space string theory $\left(p / \lambda^{1 / 4} \gg 1\right)$. The latter governed by string minimal surfaces as studied by Gross and Mende [24].

When $p=O\left(\lambda^{1 / 2}\right)$ the minimal surface is big, occupying a finite fraction of the AdS space. Intuitively, the angular momentum of the string, which is of order $p$, is comparable to the string tension $\lambda^{1 / 2}$, thus the fight between centripetal forces and tension leads to a big macroscopic string. Decreasing $p$ the string tension starts to win and shrinks the string. Thus, as we approach $p=O\left(\lambda^{1 / 4}\right)$ we expect the various strings to become pointline particles flying from the AdS boundary towards a small interaction region in AdS, as represented in the middle panel in the bottom row of figure 1 . These four almost point-like strings will then enter the small interaction region with very large energy. Interactions there are described by high energy (at fixed angle) string theory as studied by GrossMende [24], and the leading contribution to the string amplitude will now be given by a flat space minimal area.

A direct study of the AdS minimal area is hard, due to the non-linearities on the worldsheet, but in recent years an integrability based technology has been developed precisely for computing the action of minimal surfaces without ever finding the shape of these surfaces. This is fully developed for Scattering Amplitudes/Wilson loops [58-60] and three point functions of local operators [61-65]. In the case of four-point functions, the work of [66] has shown how to determine the action of the AdS minimal surfaces when the four points are aligned along a single line in the AdS boundary. Generalising this result would lead to the AdS minimal area we are looking for. A complementary question to ask is how 
the minimal area opens from the flat configuration at $p=O\left(\lambda^{1 / 4}\right)$ up to occupy a finite fraction of the AdS space when $p=O\left(\lambda^{1 / 2}\right)$. We hope to report soon on these problems and determine the full minimal areas depicted in the corner of figure 1.

In this context we would like to make a few comments on some very interesting correlation function developments in $\mathcal{N}=4 \mathrm{SYM}$ arising from Integrability explorations and commonly known as the octagon correlation functions. The octagon is a four-point correlator of very large external weights computed by Frank Coronado in perturbation theory [26] by using the hexagonalization technology [28, 29], re-derived through a boostrap program [27], recast as a compact infinite dimensional determinant in [30] and compactly described as a solution to a simple set of integro-differential equations in [36, 37]. In [31] it was shown how the octagon also computes the full non-planar expansion of particular four point correlation functions of large weights $p$ provided we scale $p \sim \sqrt{N}$ (which is of course much larger than anything discussed so far in this paper). At strong coupling the octagon was studied in [35, 37, 38]. In [35] it was shown that the hexagon exponentiates at strong coupling and it was conjectured that the exponent is nothing but the associated minimal area. So far this area was not independently computed from the worldsheet. In $[37,38]$ Belitsky and Korchemsky beautifull understood how to systematically extract any term in the strong coupling expansion.

Would be fantastic to compare those strong coupling results with our SUGRA extrapolations. As of now that is not possible for a few reasons which would be very interesting to overcome. The first reason concerns the fact the octagon is related to a correlator whose external weights are very large, much larger than anything considered in this paper and in particular much larger than $\lambda^{1 / 4}$ which is as far as we can confidently reach starting from SUGRA. Would be very interesting to compute the associated minimal area for the octagon with weights $p \sim \lambda^{1 / 2}$ and take $p / \lambda^{1 / 2}$ to be very small to hopefully interpolate between AdS minimal areas and the Gross-Mende flat space areas alluded to in this work. The second reason is more trivial: the octagon corresponds to a specific polarisation of the single-trace correlator on the $S^{5}$, i.e. it corresponds to the dynamics of a particular propagator structure, thus it is not quite the amplitude $\mathcal{A}$ we studied. Would be interesting to repeat the analysis in this work for correlators with fixed polarizations instead of fixed $R$-charge cross-ratios.

Let us nonetheless note that there is a very nice aspect of the SUGRA results which is Taylor made to allow for a smooth interpolation with the octagon strong coupling findings. One aspect of the strong coupling expansion is the scaling with $\sqrt{\lambda}$ of the correlator, which follows from the simple universal $A_{1}=1$ result of $[36,37]$ once we put together the results

$$
\begin{aligned}
\left.\left\langle T_{p} T_{p} T_{p} T_{p}\right\rangle\right|_{\text {octagon config }} & \sim[\mathbb{O}(U, V)]^{2} \\
\mathbb{O}(U, V) & =(\sqrt{\lambda})^{A_{1}^{2} / 2} e^{-g A_{0}(U, V)+B+O(1 / g)} ; \quad A_{1}=1 .
\end{aligned}
$$

We match this scaling by considering the universal scaling with $p$ which follows from our master relation (1.11), namely

$$
\lim _{p \rightarrow \infty} \mathcal{A}_{\vec{p}}(U, V, \tilde{U}, \tilde{V})=\frac{4 \pi^{4}}{(U V \tilde{U} \tilde{V})^{\frac{1}{4}}} \frac{(1+\sqrt{\tilde{U}}+\sqrt{\tilde{V}})^{2(p-2)+\frac{3}{2}}}{(1+\sqrt{U}+\sqrt{V})^{2(p+2)-\frac{3}{2}}} \times \frac{p^{4}}{\mathbf{s}_{\mathrm{cl}} \mathbf{t}_{\mathrm{cl}} \mathbf{u}_{\mathrm{cl}}}\left[1+O\left(1 / N^{2}, \lambda^{-1 / 2}\right)\right] .
$$


Notice now that $\mathbf{s}_{\mathrm{cl}}$ and $\mathbf{t}_{\mathrm{cl}}$ in (1.9)-(1.10), are function of cross ratios, but scale linearly with $p$. Thus the overall scaling of the amplitude goes with $p$. In the regime $p=O(\sqrt{\lambda})$ we recover precisely the scaling of the octagon.

It would also be fascinating to compare our findings with the octagon results of [31] where the octagon shows up as a building block in a completely re-summed large $N$ correlation function. One obstacle here pertains to the difference between single-trace operators (used in [31]) and single-particle operators (which we are using here). In particular, our connected correlator is supposed to be exponentially small (as arising from the geodesic propagation) at any order of the $1 / N$ expansion while the octagon correlator studied in [31] goes to a constant result as $\lambda \rightarrow \infty$. We believe this difference is precisely accounted by the change of basis we just mentioned and look forward to investigating this further with Till Bargheer and Frank Coronado.

The regimes of $p$ in which $p / \lambda^{1 / 4} \ll 1$ are regimes in which SUGRA, with higher derivative corrections coming from string theory, provides a valid low energy descriptions of the physics. In this framework, our large $p$ limit has unveiled a piece of the greater puzzle offered by figure 1. We started from the Mellin description of the correlators [10], and we improved that by introducing the $A d S_{5} \times S^{5}$ four-fold representation, built around $\Gamma_{\otimes}$, such that all Mellin variables (and cross ratios) are treated equally. We then took the large $p$ limit and we identified two types of contributions. From $\Gamma_{\otimes}$ we found the saddle point configuration of four geodesics shooting towards a common bulk point. From the bulk Mellin amplitude we got the IIB S-matrix in flat space. Furthermore we understood the identification between geometric data and Mandelstam invariants of the approximately ten-dimensional physics at the bulk point.

Then, the large $p$ limit guided us towards a more precise understanding of the full $A d S_{5} \times S^{5}$ amplitude. In particular, we understood how to covariantise the flat space limit a la Penendones, and how to stratify the Mellin amplitude in a large $p$ expansion. We demonstrated these two points by studying the Virasoro-Shapiro and the one-loop amplitudes, i.e. all the data currently available. For the VS amplitude we pointed out additional simplicity of the known results. At one-loop we have constructed a new finite Mellin amplitude, in correspondence with the position space bootstrap of [12-16]. Our findings put in place a more general scheme for studying the $A d S_{5} \times S^{5}$ correlators in the future.

The equality between the 10d flat space VS amplitude and the large $p$ limit of the $A d S_{5} \times S^{5}$ genus zero amplitude, which is largely unknown, is the first aspect of the large $p$ limit we worked out. Understanding a similar statement at one-loop, i.e. going beyond the 10d box in SUGRA by adding one-loop stringy corrections would be highly desirable. These corrections are known in the form of stringy integrals and it would be fascinating to connect those integrals with the Mellin space representation of the amplitude, directly. This line of thoughts goes along a similar reinterpretation of classic stringy computations under the light of new localisation results [45, 47]. In this sense, it would be even more intriguing to make contact with the generating techniques for closed and open string amplitudes recently developed in $[69,70]$.

A number of results are now available at tree level on $A d S \times S$ spaces. The case of $A d S_{3} \times S^{3}$ [71-73] is likely to enjoy hidden-symmetric properties analogous to $A d S_{5} \times S^{5}$, 
and it would be a natural place where to explore the large $p$ limit in the way we did in this paper. At one loop the six-dimensional box is even simpler, and it could be the right starting point for bootstrapping the $A d S_{3} \times S^{3}$ one-loop amplitudes.

Very recently, the authors of $[74,75]$ conjectured all tree level amplitudes for the maximally symmetric theories in $d=3$ and $d=6$. Amplitudes in these dimensions are more likely to be attached to the various propagator structures, since it is yet unclear what superconformal symmetry manifests into - for example long superconformal blocks do not show any kinematic common factor - However, as we discussed in this paper, we expect all fundamental properties of flat M-theory to emerge in the large $p$ limit. As an anticipation of this expectation, $[74,75]$ pointed out the emerging of nice features in the flat space limit a la Penedones, specifically in what they call the R-symmetry polynomial in combination with the universal 1/stu term, which appears to be the same as in $A d S_{5} \times S^{5}$. As we learned in this paper, this is the starting point towards the large $p$ limit.

Kid A and kid B want to dig a big solid tunnel in the beach. Each one starts digging a tunnel entrance. They seem so far at first. They dig and dig and at some point they feel each other's finger tips, barely touching as they scratch the sand digging from either side. They stand and look through each tunnel entrance to see a beautiful tiny light ray coming from the other side. The kids smile. At this point they know they will succeed. It is just a matter of digging carefully and steadily. Kid A is Integrability. Kid B is SUGRA.

\section{Acknowledgments}

We would like thank Till Bargheer, Nathan Berkovits, Frank Coronado, Vasco Goncalves, Andrea Guerrieri, Shota Komatsu, Joao Penedones, Amit Sever for several enlightening discussions. FA would like to thank James Drummond and Paul Heslop, Hynek Paul and Michele Santagata, for collaboration on related topics, and especially James Drummond for pointing out ref. [53] in the 2017. FA acknowledges the ICTP South American Institute for Fundamental Research (ICTP-SAIFR) for hospitality and financial support during the period this work started. FA is partially supported by the ERC-STG grant 637844HBQFTNCER. Research at the Perimeter Institute is supported in part by the Government of Canada through NSERC and by the Province of Ontario through MRI. This work was additionally supported by a grant from the Simons Foundation (PV: \#488661) and FAPESP grants 2016/01343-7 and 2017/03303-1.

\section{A Holographic correlators and the large $p$ limit}

\section{A.1 Conventions on Mellin and Mandelstam variables}

We will define first the Mellin transform of a generic four-point function,

$$
\left\langle\mathcal{O}_{p_{1}}\left(x_{1}\right) \mathcal{O}_{p_{2}}\left(x_{2}\right) \mathcal{O}_{p_{3}}\left(x_{3}\right) \mathcal{O}_{p_{4}}\left(x_{4}\right)\right\rangle=\oint \mathcal{M}\left(c_{\alpha \beta}\right) \prod_{1 \leq i<j \leq 4}^{4} \frac{\Gamma\left[c_{\alpha \beta}\right]}{\left(x_{i}-x_{j}\right)^{2 c_{\alpha \beta}}}
$$


The Symanzik variables $c_{\alpha \beta}=c_{\beta, \alpha}\left(\right.$ with $\left.c_{\alpha \alpha}=0\right)$ are constrained requiring

$$
\sum_{\alpha \neq \beta} c_{\alpha \beta}=p_{\beta}
$$

and can be solved by analogy with Mandelstam variables, by defining $c_{\alpha \beta}=c_{\alpha \beta}(s, t, \vec{p})$, where the integration variables are $s$, and $t$, and a single constraint gives $u$. In Mellin space it is then natural to have $-s=c_{12}$ to appear in the argument of the $\Gamma$ functions, similarly for $t$ and $u$.

The S-matrix for massless scattering is by construction a function only of the physical Mandelstam invariants, and since we are considering a $2 \rightarrow 2$ process, we find $\mathcal{S}^{\text {flat }}=$ $\mathcal{S}^{\text {flat }}\left(k_{s}, k_{t}\right)$ where

$$
k_{s}=-\left(k_{1}+k_{2}\right)^{2} ; \quad k_{t}=-\left(k_{1}+k_{4}\right)^{2} ; \quad k_{u}=-\left(k_{1}+k_{3}\right)^{2} .
$$

The quantities $k_{s}$ and $k_{t}$ are the physical $s$ and $t$ Mandelstam invariants, but these are not the same as the Mellin space variables. In the above conventions, the flat space formula of Penedones [5], reads,

$$
\mathcal{M}(s, t)=C \int_{0}^{\infty} d \beta \beta^{\Sigma-\frac{d}{2}-1} e^{-\beta} \mathcal{S}^{\text {flat }}\left(k_{s}=\frac{4 \beta}{R^{2}} s, k_{t}=\frac{4 \beta}{R^{2}} t\right) .
$$

To compare with [5] notice that $\delta_{i j}^{\text {there }}=c_{\alpha \beta}^{\text {here }}$ and $s_{i j}^{\text {there }}$ scales like $2 s^{\text {here }}$.

\section{A.2 Conventions on SUGRA amplitudes}

Four-point functions of half-BPS operators admit a splitting into free and dynamical contributions, according to the partial-non-renormalisation theorem [39], as we now review.

Free theory four-point correlators are given by a sum over propagator structures weighted by the corresponding color factor $a_{\gamma, k}$. Without loss of generality we consider $p_{43} \geq p_{21} \geq 0$, and write the free theory contribution as

$$
\left\langle\mathcal{O}_{p_{1}}\left(x_{1}\right) \ldots \mathcal{O}_{p_{4}}\left(x_{4}\right)\right\rangle_{\text {free }}=\mathcal{P}\left(\left\{g_{i j}\right\}\right) \sum_{\substack{p_{43} \leq \gamma_{12} \\ 0 \leq \gamma_{23} \leq \gamma_{12}}} a_{\gamma_{12}, \gamma_{23}}\left(\frac{g_{13} g_{24}}{g_{12} g_{34}}\right)^{\frac{1}{2}\left(\gamma_{12}-p_{3}-p_{4}\right)}\left(\frac{g_{14} g_{23}}{g_{13} g_{24}}\right)^{\gamma_{23}}
$$

where

$$
\mathcal{P}=g_{12}^{\frac{p_{1}+p_{2}-p_{3}-p_{4}}{2}} g_{14}^{\frac{p_{1}+p_{4}-p_{3}-p_{2}}{2}} g_{24}^{\frac{p_{2}+p_{4}-p_{1}-p_{3}}{2}}\left(g_{13} g_{24}\right)^{p_{3}} .
$$

Here $\gamma_{12}$ counts the number of bridges going from $\mathcal{O}_{p_{1}}\left(x_{1}\right) \mathcal{O}_{p_{2}}\left(x_{2}\right)$ to $\mathcal{O}_{p_{3}}\left(x_{1}\right) \mathcal{O}_{p_{4}}\left(x_{2}\right)$. The first diagram, i.e. $\gamma_{12}=p_{43}$ with $\gamma_{23}=0$, is the diagram where all bridges of $\mathcal{O}_{p_{3}}$ link with $\mathcal{O}_{p_{4}}$, therefore there are $p_{43}$ bridges going from $\mathcal{O}_{p_{4}}$ to the pair $\mathcal{O}_{p_{1}} \mathcal{O}_{p_{2}}$.

Cross ratios will be parametrised as follows,

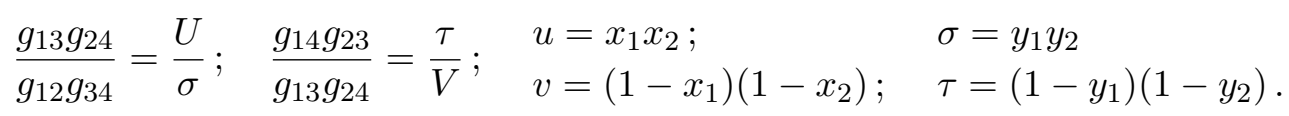


The variables $\sigma$ and $\tau$ are those coming from analytic superspace (see [67] for a more recent discussion). We will start from these, since it is useful to match conventions with previous literature, and we will then introduce our $\tilde{U}=\sigma$ and $\tilde{V}=\tau$.

The dynamical contribution inherits from superconformal symmetry a specific structure [39],

$$
\left\langle\mathcal{O}_{p}\left(x_{1}\right) \ldots \mathcal{O}_{p}\left(x_{4}\right)\right\rangle_{\text {dynamical }}=\prod_{1 \leq i, j \leq 2}\left(x_{i}-y_{j}\right) \times \mathcal{P}\left(\left\{g_{i j}\right\}\right) \times \mathcal{D}_{\vec{p}}(U, V, \sigma, \tau) .
$$

In the strong 't Hooft coupling regime, the theory lives on a classical $A d S_{5} \times S^{5}$ and the quantum corrections are organised as a double expansion in $1 / N^{2}$ and $\lambda^{-1 / 2}$. In particular, the dynamical function scales like a connected correlator. In this regime we define the amplitude $\mathcal{A}_{\vec{p}}$ of the correlator as

$$
\mathcal{D}_{\vec{p}}(U, V, \sigma, \tau)=\frac{p_{1} p_{2} p_{3} p_{4}}{N^{2}} \mathcal{A}_{\vec{p}}(U, V, \sigma, \tau) .
$$

The amplitude $\mathcal{A}$ encodes all the non-trivial information about the dynamics and can be written as a double integral and a double sum,

$$
\mathcal{A}_{\vec{p}}(u, v, \sigma, \tau) \equiv \iint d s d t u^{s} v^{t} \sum_{T} \sigma^{-i-j+p_{3}-2} \tau^{j} \times \Gamma(s, t, i, j) \times \mathcal{M}_{\vec{p}}(s, t, i, j)
$$

where

$$
\begin{aligned}
\Gamma(s, t, i, j) & =\frac{\Gamma[-s] \Gamma[-t] \Gamma[-u] \Gamma\left[-s+c_{s}\right] \Gamma\left[-t+c_{t}\right] \Gamma\left[-u+c_{u}\right]}{k ! j ! i !\left(k+c_{s}\right) !\left(j+c_{t}\right) !\left(i+c_{u}\right) !} \\
c_{s} & =\frac{p_{1}+p_{2}-p_{3}-p_{4}}{2} ; \quad c_{t}=\frac{p_{1}+p_{4}-p_{2}-p_{3}}{2} ; \quad c_{u}=\frac{p_{2}+p_{4}-p_{3}-p_{1}}{2} .
\end{aligned}
$$

The definition of $k$ follows from the relation $i+j+k=p_{3}-2$. The definition of $u$ is

$$
s+t+u=-p_{3}-2 .
$$

The double sum over $i$ and $j$ runs over the set of integers,

$$
T=\{i \geq 0 ; j \geq 0 ; i+j \leq \kappa-2\} \quad \kappa=\frac{1}{2}\left(\min \left(p_{1}+p_{2}, p_{3}+p_{4}\right)-p_{43}\right)
$$

where $\kappa$ is the degree of extremality. Notice that $\kappa=\min \left(c_{s}, 0\right)+p_{3}$, therefore $i+j \leq \kappa-2$ iff $k \geq \max \left(-c_{s}, 0\right)$, and $k !\left(k+c_{s}\right)$ ! is well defined. In our conventions $c_{u}, c_{t} \geq 0$ but $c_{s}$ can have both signs. In the main text we changed variables from $i$ and $j$ to the new variables

$$
\tilde{s}=-i-j+p_{3}-2 ; \quad \tilde{t}=j ; \quad \tilde{s}+\tilde{t}+\tilde{u}=p_{3}-2 .
$$

In these new variables $s$ is aligned with $\tilde{s}$ and the gamma factor becomes

$$
\Gamma(s, t, \tilde{s}, \tilde{t})=\frac{\Gamma[-s] \Gamma[-t] \Gamma[-u] \Gamma\left[-s+c_{s}\right] \Gamma\left[-t+c_{t}\right] \Gamma\left[-u+c_{u}\right]}{\Gamma[1+\tilde{s}] \Gamma[1+\tilde{t}] \Gamma[1+\tilde{u}] \Gamma\left[1+\tilde{s}+c_{s}\right] \Gamma\left[1+\tilde{t}+c_{t}\right] \Gamma\left[1+\tilde{u}+c_{u}\right]} .
$$


The triangle is now

$$
T=\left\{\tilde{s} \geq \max \left(0,-\frac{1}{2}\left(p_{1}+p_{2}-p_{3}-p_{4}\right)\right) ; \tilde{t} \geq 0 ; \tilde{u} \geq 0\right\} .
$$

The two cases of $\tilde{s}$ have to do with the two possible inequalities $c_{s} \geq s \geq 0$ or $\tilde{s} \geq-c_{s} \geq 0$, which depend on $\max \left(p_{1}+p_{2}, p_{3}+p_{4}\right)$. This is the only freedom left in our conventions.

The four-fold representation of $\mathcal{A}$ in (A.9) is obtained by turning the discrete sum over $T$ into a Mellin integral over $\tilde{s}$ and $\tilde{t}$. The inequalities which define $T$ are implemented by the positivity of the $\Gamma$ functions in the denominator of $\Gamma(s, t, \tilde{s}, \tilde{t})$, and thus we only need to pick a domain where to insert simple poles. There are various choices, and here we consider

$$
\mathcal{A}_{\vec{p}}(U, V, \tilde{U}, \tilde{V})=\iint d s d t \iint d \tilde{s} d \tilde{t} U^{s} V^{t} \tilde{U}^{\tilde{s}} \tilde{V}^{\tilde{t}} \times \Gamma_{\otimes} \times \mathcal{M}_{\vec{p}}(s, t, \tilde{s}, \tilde{t})
$$

where now

$$
\begin{aligned}
\Gamma_{\otimes} & =\mathfrak{S} \frac{\Gamma[-s] \Gamma[-t] \Gamma[-u] \Gamma\left[-s+c_{s}\right] \Gamma\left[-t+c_{t}\right] \Gamma\left[-u+c_{u}\right]}{\Gamma[1+\tilde{s}] \Gamma[1+\tilde{t}] \Gamma[1+\tilde{u}] \Gamma\left[1+\tilde{s}+c_{s}\right] \Gamma\left[1+\tilde{t}+c_{t}\right] \Gamma\left[1+\tilde{u}+c_{u}\right]} \\
\mathfrak{S} & =\pi^{2} \frac{(-)^{\tilde{t}}(-)^{\tilde{u}}}{\sin (\pi \tilde{t}) \sin (\pi \tilde{u})} .
\end{aligned}
$$

In the four-fold representation above we turned to the more appropriate $\sigma=\tilde{U}$ and $\tau=\tilde{V}$.

Below we discuss the relation between poles in $\Gamma_{\otimes}$ and the OPE expansion. First, notice that double poles of $s$ and $t$ occur respectively for $s \geq \max \left(0, c_{s}\right)$, i.e. $U^{\max \left(0, c_{s}\right)}$, and $t \geq c_{t} \geq 0$.

\section{A.3 OPE view on the Mellin amplitude}

Shifting the AdS Mellin variables to align double poles in $s$ and $t$ to lie on the positive real axis, we find that the $\log ^{1} u$ discontinuity of the dynamical function has a Taylor expansion in small $u$ and small $v$ starting with leading powers ${ }^{21}$

$$
\left.U^{\frac{p_{3}+p_{4}}{2}} \mathcal{D}_{p_{1} p_{2} p_{3} p_{4}}^{(1)}(U, V, \tilde{U}, \tilde{V})\right|_{\log ^{1} u}=U^{\frac{\max \left(p_{1}+p_{2}, p_{3}+p_{4}\right)}{2}} V^{\frac{p_{43}-p_{21}}{2}}\left(\mathcal{A}_{0,0}(\tilde{U}, \tilde{V})+\ldots\right)
$$

The value of $\max \left(p_{1}+p_{2}, p_{3}+p_{4}\right)$ is the threshold twist for exchange of long two-particle operators in the common $\operatorname{OPE}\left(\mathcal{O}_{p_{1}} \times \mathcal{O}_{p_{2}}\right) \cap\left(\mathcal{O}_{p_{3}} \times \mathcal{O}_{p_{4}}\right)$. The power of $V$ follows from crossing. To see this threshold twist consider the behaviour of the three point functions of two external single-particle operators $\mathcal{O}_{p_{i}} \mathcal{O}_{p_{j}}$ with a two-particle operator $\mathcal{K}$ of twist $\tau$ in the rep $[a b a]$ of $s u(4)$, as function of the twist $\tau$

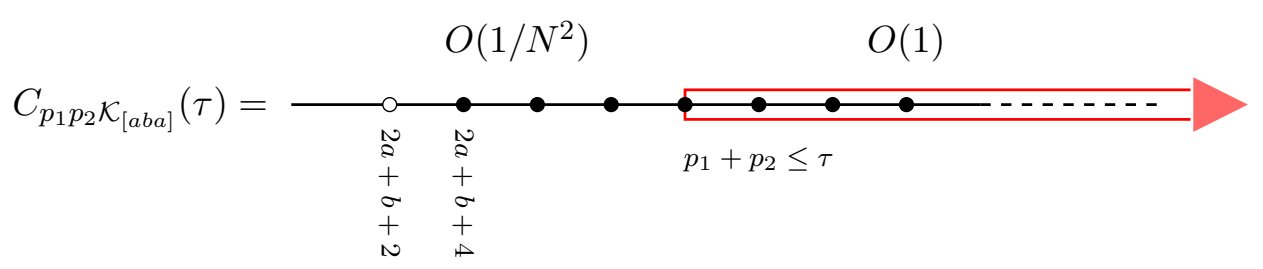

${ }^{21}$ The 1.h.s. normalisation $U^{\frac{p_{43}}{2}+p_{3}}$ is due to the form of the superblocks, and the prefactor in (A.7). 
In the four point function we need to put this line together with the analogous line for

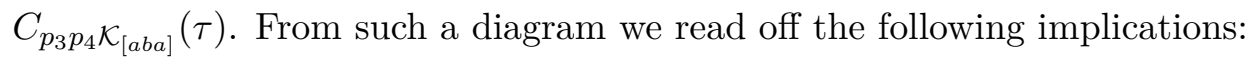

1. Two particle operators with twist above the threshold have an $O(1)$ three point functions in disconnected free theory.

2. Long two-particle operators acquire a $1 / N^{2}$ anomalous dimension [16] and have an $O(1)$ three point functions in disconnected free theory. Therefore they build up the leading logarithmic discontinuity (A.19), when they are exchanged at tree level, above threshold.

3. Other CFT data can be extracted from the window $\min \left(p_{1}+p_{2}, p_{3}+p_{4}\right) \leq \tau<$ $\max \left(p_{1}+p_{2}, p_{3}+p_{4}\right)$.

4. The below-window region is bounded by the unitarity bound, which for a given rep $[a b a]$ of $s u(4)$ is $\tau_{[a b a]}=2 a+b+2$. The first contributions below window are of order $1 / N^{4}$.

In fact, simple poles in $s$ and $t$ in $\mathcal{M}^{(1)}=1 /(\mathbf{s}+1) /(\mathbf{t}+1) /(\mathbf{u}+1)$ are known to contribute precisely at the unitarity bound $\tau_{[a b a]}=2 a+b+2$, and cancel the corresponding contribution coming from connected free theory, in the basis of superblock [16, 21, 22]. We see now that all these simple poles correspond to the locus $\mathbf{s}=-1$ and $\mathbf{t}=-1$ projected onto the planes $s$ and $t$, for fixed $\tilde{s}$ and $\tilde{t}$. More precisely, a point $(\tilde{s}, \tilde{t})$ in the triangle $T$,

$$
T=\left\{\tilde{t} \geq 0 ; \tilde{u} \geq 0 ; \tilde{t}+\tilde{u} \leq \min \left(0, c_{s}\right)+p_{3}-2\right\} ; \quad \tilde{s}+\tilde{t}+\tilde{u}=p_{3}-2
$$

gives a simple pole in $s$, from $\mathbf{s}=-1$, which corresponds to a contribution at twist $\tau=$ $p_{3}+p_{4}-2-2 \tilde{s}=p_{43}+2+2(\tilde{t}+\tilde{u})$. In the second equality, we used the relation in (A.20). Equating this value to $\tau_{[a b a]}$ we find that all possible contributions at the unitarity bound $\tau=\tau_{[a b a]}$ are filled in

$$
\tau_{[a b a]}=2(\tilde{t}+\tilde{u})+2+p_{43} \quad \leftrightarrow \quad a+\frac{1}{2}\left(b-p_{43}\right)=\tilde{t}+\tilde{u} \in T
$$

because $a=0, \ldots \kappa-2$ and $b=p_{43}, \ldots, p_{43}+2(\kappa-2)-2 a$, with $\kappa=\min \left(0, c_{s}\right)+p_{3}$ the degree of extremality we already encountered.

\section{B Mellin saddle point details}

\section{B.1 Saddle point on $\operatorname{AdS}_{5}$}

In the supergravity regime the Mellin amplitude does not exponentiate and the saddle factorises into $A d S_{5}$ and the $S^{5}$ contribution, since it only comes from $\Gamma_{\otimes}$.

Let us begin from $A d S_{5}$ saddle. Matching the arguments of $\Gamma_{\otimes}$, we define

$$
\mathcal{H}_{A d S_{5}}(s, t, \vec{p})=U^{s} V^{t} \prod_{1 \leq \alpha<\beta \leq 4} \Gamma\left[c_{\alpha \beta}\left(s, t, u, p_{i}+2\right)\right]
$$

where $\sum_{\alpha=1}^{4} c_{\alpha \beta}\left(s, t, p_{i}\right)=p_{\beta}$ are Symanzik variables. Written in this fashion, the study of the $A d S_{5}$ saddle is a $\bar{D}$ generalisation of a classic study for hypergeometrics [68]. 
The large $p$ limit is taken by rescaling $\left\{s, t, p_{i=1,2,3,4}\right\}=n\left\{s^{*}, t^{*}, d_{i=1,2,3,4}\right\}$ and letting $n \rightarrow \infty$. The limiting behaviour of the Symanzik variables has the following form

$$
\begin{aligned}
c_{\alpha \beta}\left(s, t, p_{i}+2\right) & \rightarrow n c_{\alpha \beta}\left(s^{*}, t^{*}, d_{i}\right)+r_{\alpha \beta} \\
\Gamma\left[c_{\alpha \beta}\left(s, t, p_{i}+2\right)\right] & \rightarrow \sqrt{2 \pi} e^{-n c_{\alpha \beta}}\left(n c_{\alpha \beta}\right)^{n c_{\alpha \beta}+r_{\alpha \beta}-1 / 2} .
\end{aligned}
$$

Notice that $u^{*}=-s^{*}-t^{*}-q$ and there are only two non vanishing $r_{\alpha \beta}$, with value +2 , i.e. those taking into account the difference $-u+u^{*}$. The limit of $\mathcal{H}_{A d S_{5}}$ is then

$$
\begin{aligned}
\mathcal{H}_{A d S_{5}}(s, t, \vec{p}) \rightarrow & \frac{(2 \pi)^{3}}{n^{3}}\left(\prod_{\alpha<\beta} n^{r_{\alpha \beta}}\right) e^{-\frac{p_{1}+p_{2}+p_{3}+p_{4}}{2}} n^{\frac{p_{1}+p_{2}+p_{3}+p_{4}}{2}} \times \\
& \oint\left(n^{2} d s^{*} d t^{*}\right) \prod_{\alpha<\beta} c_{\alpha \beta}^{r_{\alpha \beta}-\frac{1}{2}}\left(s^{*}, t^{*}, d_{i}\right) \exp \left[-n S_{A d S_{5}}\right]
\end{aligned}
$$

where the effective action reads,

$$
S_{A d S_{5}}=-s^{*} \log (U)-t^{*} \log (V)-\sum_{1 \leq \alpha<\beta \leq 4} c_{\alpha \beta}\left(s^{*}, t^{*}, d_{i}\right) \log \left[c_{\alpha \beta}\left(s^{*}, t^{*}, d_{i}\right)\right] .
$$

The saddle point equations are

$$
\frac{\left(-s^{*}\right)\left(+\frac{d_{1}+d_{2}-d_{3}-d_{4}}{2}-s^{*}\right)}{\left(-u^{*}\right)\left(+\frac{d_{2}+d_{4}-d_{1}-d_{3}}{2}-u^{*}\right)}=U ; \quad \frac{\left(-t^{*}\right)\left(+\frac{d_{1}-d_{2}-d_{3}+d_{4}}{2}-t^{*}\right)}{\left(-u^{*}\right)\left(+\frac{d_{2}+d_{4}-d_{1}-d_{3}}{2}-u^{*}\right)}=V .
$$

Recall that the saddle point should be such that the arguments of the log are positive, i.e. the saddle point should stay away from the accumulation of the gamma function poles in the large $p$ limit.

We can rewrite the Effective Action as

$$
\begin{aligned}
S_{A d S_{5}}= & +s^{*}\left(\log \left[\frac{\left(-s^{*}\right)\left(c_{s}-s^{*}\right)}{\left(-u^{*}\right)\left(c_{u}-u^{*}\right)}\right]-\log (U)\right)+t^{*}\left(\log \left[\frac{\left(-t^{*}\right)\left(c_{t}-t^{*}\right)}{\left(-u^{*}\right)\left(c_{u}-u^{*}\right)}\right]-\log (V)\right) \\
& -\left(S_{A d S_{5}}^{0} \equiv \sum_{1 \leq \alpha<\beta \leq 4} c_{\alpha \beta}\left(0,0, d_{i}\right) \log \left[c_{\alpha \beta}\left(s^{*}, t^{*}, d_{i}\right)\right]\right) .
\end{aligned}
$$

When the external charges are all equal, we can take the square root of equations (B.5). The solution is then

$$
-s_{\mathrm{cl}}=d \frac{\sqrt{U}}{1+\sqrt{U}+\sqrt{V}} ; \quad-t_{\mathrm{cl}}=d \frac{\sqrt{V}}{1+\sqrt{U}+\sqrt{V}} .
$$

In order to compute $\mathcal{H}_{A d S_{5}}(u, v, p p p p)$ at the saddle, we then need to evaluate both $\mathcal{S}_{A d S_{5}}^{0}$ and the quadratic fluctuations $\mathcal{S}_{A d S_{5}}^{\prime \prime}$. For the action on-shell we find

$$
-n S_{A d S_{5}}^{0}(u, v, p p p p)=2 p \log \left(d+s_{\mathrm{cl}}+t_{\mathrm{cl}}\right)=+2 p \log \left(\frac{d}{1+\sqrt{U}+\sqrt{V}}\right)
$$


and for the Hessian

$$
\operatorname{det} S_{A d S_{5}}^{\prime \prime}\left(s^{*}, t^{*}, p p p p\right)=\operatorname{det}\left(\begin{array}{cc}
-\frac{2}{s^{*}}-\frac{2}{u^{*}} & -\frac{2}{u^{*}} \\
-\frac{2}{u^{*}} & -\frac{2}{t^{*}}-\frac{2}{u^{*}}
\end{array}\right)=-\frac{4 d}{s^{*} t^{*} u^{*}} .
$$

We will have to include $\frac{2 \pi}{n}\left|\operatorname{det} \mathcal{S}_{A d S_{5}}^{\prime \prime}\right|^{-\frac{1}{2}}$.

Finally, the saddle point approximation gives

$$
\left.\mathcal{H}_{A d S_{5}}(u, v, p p p p)\right|_{\text {saddle approx }}=\frac{(2 \pi p)^{4}}{2 p^{2}} \frac{e^{-2 p} p^{2 p}}{(1+\sqrt{U}+\sqrt{V})^{2(p+2)}}\left[\frac{d^{3 / 2}}{\sqrt{\left(d+s_{\mathrm{cl}}+t_{\mathrm{cl}}\right) s_{\mathrm{cl}} t_{\mathrm{cl}}}}\right] \text {. }
$$

In the last line we have made crossing symmetry manifest, i.e. we extracted a term which has the right crossing transformation, in blue, and put the remaining (crossing invariant) terms together. The crossing transformations are

$$
\mathcal{H}_{A d S_{5}}(U, V, p p p p)=\frac{1}{V^{p+2}} \mathcal{H}_{A d S_{5}}\left(\frac{U}{V}, \frac{1}{V}, p p p p\right)=\mathcal{H}_{A d S_{5}}(V, U, p p p p)
$$

and the crossing invariant term is

$$
\frac{d^{3 / 2}}{\sqrt{\left(d+s_{\mathrm{cl}}+t_{\mathrm{cl}}\right) s_{\mathrm{cl}} t_{\mathrm{cl}}}}=\frac{(1+\sqrt{U}+\sqrt{V})^{3 / 2}}{(U V)^{1 / 4}} .
$$

Both $U \leftrightarrow V$ and $U \rightarrow U / V, V \rightarrow 1 / V$, leave (B.12) invariant.

By writing $\mathcal{H}_{A d S_{5}}(U, V, p p p p)$ in the form (B.10) we should interpret the contribution above as counting for the total gaussian fluctuation around the saddle point. In fact, (B.12) is the combined result from the Hessian and $\prod_{\alpha<\beta} c_{\alpha \beta}^{-1 / 2}\left(s_{\mathrm{cl}}, t_{\mathrm{cl}}, d\right)$.

\section{B.2 Saddle point on $S^{5}$}

The study of the saddle on the sphere follows similar steps. Define from $\Gamma_{\otimes}$,

$$
\mathcal{H}_{S^{5}}(\tilde{s}, \tilde{t}, \vec{p})=\tilde{U}^{\tilde{s}} \tilde{V}^{\tilde{t}}\left[\mathfrak{S}=\pi^{2} \frac{(-)^{\tilde{u}}(-)^{\tilde{t}}}{\sin (\pi \tilde{u}) \sin (\pi \tilde{t})}\right] \prod_{1 \leq \alpha<\beta \leq 4} \frac{1}{\Gamma\left[1+C_{\alpha \beta}\left(\tilde{s}, \tilde{t}, p_{i}-2\right)\right]}
$$

which includes the factor $\mathfrak{S}$ and where again we have introduce Symanzik variables $C_{\alpha \beta}$ such that $\sum_{\alpha} C_{\alpha \beta}=p_{\beta}$.

In the large $p$ limit take $\left\{\tilde{s}, \tilde{t}, p_{i=1,2,3,4}\right\}=n\left\{\tilde{s}^{*}, \tilde{t}^{*}, d_{i=1,2,3,4}\right\}, \tilde{u}^{*}=-\tilde{s}^{*}-\tilde{t}^{*}+d_{3}$, and let $n \rightarrow \infty$. We find

$$
\begin{aligned}
C_{\alpha \beta}\left(\tilde{s}, \tilde{t}, p_{i}-2\right) & \rightarrow n C_{\alpha \beta}\left(\tilde{s}^{*}, \tilde{t}^{*}, d_{i}\right)+R_{\alpha \beta} \\
1 / \Gamma\left[1+C_{\alpha \beta}\left(s, t, p_{i}+2\right)\right] & \rightarrow 1 / \sqrt{2 \pi} e^{+n C_{\alpha \beta}}\left(n C_{\alpha \beta}\right)^{-n C_{\alpha \beta}-R_{\alpha \beta}-\frac{1}{2}}
\end{aligned}
$$

where the two non vanishing $R_{\alpha \beta}$ now have values -2 . Notice also that $\frac{(-)^{\tilde{u}}(-)^{\tilde{t}}}{\sin (\pi \tilde{u}) \sin (\pi \tilde{t})} \rightarrow 4$. From the asymptotics

$$
\begin{aligned}
\mathcal{H}_{S^{5}}(\tilde{s}, \tilde{t}, \vec{p}) \rightarrow & \frac{4 \pi^{2}}{(2 \pi)^{3} n^{3}}\left(\prod_{\alpha<\beta} n^{-R_{\alpha \beta}}\right) e^{+\frac{p_{1}+p_{2}+p_{3}+p_{4}}{2}} n^{-\frac{p_{1}+p_{2}+p_{3}+p_{4}}{2}} \\
& \oint\left(n^{2} d \tilde{s}^{*} d \tilde{t}^{*}\right) \prod_{\alpha<\beta} C_{\alpha \beta}^{-R_{\alpha \beta}-\frac{1}{2}}\left(\tilde{s}^{*}, \tilde{t}^{*}, d_{i}\right) \exp \left[-n S_{S^{5}}\right] .
\end{aligned}
$$


The effective action reads

$$
S_{S^{5}}=-\tilde{s}^{*} \log (\tilde{U})-\tilde{t}^{*} \log (\tilde{V})+\sum_{1 \leq \alpha<\beta \leq 4} C_{\alpha \beta}\left(\tilde{s}^{*}, \tilde{t}^{*}, d_{i}\right) \log \left[C_{\alpha \beta}\left(\tilde{s}^{*}, \tilde{t}^{*}, d_{i}\right)\right]
$$

The saddle point equations are

$$
\frac{\left(+\tilde{s}^{*}\right)\left(c_{s}+\tilde{s}^{*}\right)}{\left(+\tilde{u}^{*}\right)\left(c_{u}+\tilde{u}^{*}\right)}=\tilde{U} ; \quad \frac{\left(+\tilde{t}^{*}\right)\left(c_{t}+\tilde{t}^{*}\right)}{(+\tilde{u})\left(c_{u}+\tilde{u}^{*}\right)}=\tilde{V}
$$

We can also rewrite the Effective Action as

$$
\begin{aligned}
S_{S_{5}}= & +\tilde{s}^{*}\left(\log \left[\frac{\left(+\tilde{s}^{*}\right)\left(c_{s}+\tilde{s}^{*}\right)}{\left(+\tilde{u}^{*}\right)\left(c_{u}+\tilde{u}^{*}\right)}\right]-\log (\tilde{U})\right)+t^{*}\left(\log \left[\frac{\left(+t^{*}\right)\left(c_{t}+\tilde{t}^{*}\right)}{\left(+\tilde{u}^{*}\right)\left(c_{u}+\tilde{u}^{*}\right)}\right]-\log (\tilde{V})\right) \\
& +\left(S_{S_{5}}^{0} \equiv \sum_{1 \leq \alpha<\beta \leq 4} C_{\alpha \beta}\left(0,0, d_{i}\right) \log \left[C_{\alpha \beta}\left(\tilde{s}^{*}, \tilde{t}^{*}, d_{i}\right)\right]\right) .
\end{aligned}
$$

The explicit solution for the case of equal charges is

$$
\tilde{s}_{\mathrm{cl}}=d \frac{\sqrt{\tilde{U}}}{1+\sqrt{\tilde{U}}+\sqrt{\tilde{V}}} ; \quad \tilde{t}_{\mathrm{cl}}=d \frac{\sqrt{\tilde{V}}}{1+\sqrt{\tilde{U}}+\sqrt{\tilde{V}}} .
$$

The action on shell then reads

$$
-n S_{S_{5}}^{0}(\tilde{U}, \tilde{V}, p p p p)=-2 p \log \left(d-\tilde{s}_{\mathrm{cl}}-\tilde{t}_{\mathrm{cl}}\right)=-2 p \log \left(\frac{d}{1+\sqrt{\tilde{U}}+\sqrt{\tilde{V}}}\right)
$$

and for the Hessian we find

$$
\operatorname{det} S_{S^{5}}^{\prime \prime}\left(S_{p p p p}^{*}, T_{p p p p}^{*}\right)=\frac{4 d}{\tilde{s}^{*} \tilde{t}^{*} \tilde{u}^{*}}
$$

Considering all the relevant contributions (we will have to include $\frac{2 \pi}{n}\left|\operatorname{det} \mathcal{S}_{S_{5}}^{\prime \prime}\right|^{-\frac{1}{2}}$ ) the saddle point approximation gives

$$
\left.\mathcal{H}_{S_{5}}(\tilde{U}, \tilde{V}, p p p p)\right|_{\text {saddle approx }}=\frac{p^{2}}{2} \frac{e^{+2 p} p^{-2 p}}{(1+\sqrt{\tilde{U}}+\sqrt{\tilde{V}})^{2(2-p)}}\left[\frac{d^{3 / 2}}{\sqrt{\left(d-\tilde{s}_{\mathrm{cl}}-\tilde{t}_{\mathrm{cl}}\right) \tilde{s}_{\mathrm{cl}} \tilde{t}_{\mathrm{cl}}}}\right] .
$$

As in the case of $A d S_{5}$, we have written the last expression by making manifest crossing symmetry, which for $\mathcal{H}_{S^{5}}$ means,

$$
\mathcal{H}_{S^{5}}(\tilde{U}, \tilde{V}, p p p p)=\tilde{V}^{p-2} \mathcal{H}_{S^{5}}\left(\frac{\tilde{U}}{\tilde{V}}, \frac{1}{\tilde{V}}, p p p p\right)=\mathcal{H}_{S^{5}}(\tilde{V}, \tilde{U}, p p p p)
$$

The blue colored term provides the correct crossing transformation, and the remaining one is crossing invariant. The latter carries the total fluctuations of the saddle configuration. 


\section{Virasoro-Shapiro}

\section{C.1 Low energy expansion and asymptotics}

The Virasoro-Shapiro amplitude in our normalisation reads [56]

$$
\mathcal{A}_{\mathrm{VS}}=\frac{1}{s t u} \frac{\Gamma\left(1-\frac{\alpha^{\prime} s}{4}\right) \Gamma\left(1-\frac{\alpha^{\prime} t}{4}\right) \Gamma\left(1-\frac{\alpha^{\prime} u}{4}\right)}{\Gamma\left(1+\frac{\alpha^{\prime} s}{4}\right) \Gamma\left(1+\frac{\alpha^{\prime} t}{4}\right) \Gamma\left(1+\frac{\alpha^{\prime} u}{4}\right)} ; \quad u=-s-t
$$

where $s=-\left(k_{1}+k_{2}\right)^{2}, t=-\left(k_{1}+k_{4}\right)^{2}$ and $u=-\left(k_{1}+k_{3}\right)^{2}$. At low energy we expand around $s, t, u=0$ to recover the famous infinite series representation

$$
\mathcal{A}_{\mathrm{VS}}=\frac{1}{s t u} \exp \left[\sum_{n \geq 1} \frac{2 \zeta_{2 n+1}}{2 n+1}\left(\frac{\alpha^{\prime}}{4}\right)^{2 n+1}\left(s^{2 n+1}+t^{2 n+1}+u^{2 n+1}\right)\right]
$$

in terms of the odd zeta functions $\zeta_{3}, \zeta_{5}, \ldots$.

A totally different expansion corner corresponds to the Gross-Mende (GM) regime of large energy at fixed angle corresponding to all Mandelstam large. In this case, we obtain another beautiful infinite series representation

$$
\begin{aligned}
& \mathcal{A}_{\mathrm{GM}}=\exp \left[-\frac{\alpha^{\prime}}{4} s \log \left(-s^{2}\right)-\frac{\alpha^{\prime}}{4} t \log \left(-t^{2}\right)-\frac{\alpha^{\prime}}{4} u \log \left(-u^{2}\right)\right] \\
& \mathcal{A}_{\mathrm{VS}}=+\frac{i}{s t u} \mathcal{A}_{\mathrm{GM}}\left[\sum_{n \geq 1} \frac{B_{2 n}}{n(2 n-1)}\left(\frac{4}{\alpha^{\prime}}\right)^{2 n-1}\left(\frac{1}{s^{2 n-1}}+\frac{1}{t^{2 n-1}}+\frac{1}{u^{2 n-1}}\right)\right]
\end{aligned}
$$

which must also be known to the experts (although we could not find it anywhere). Here $B_{n}$ are the Bernoulli numbers. The amplitude $\mathcal{A}_{\mathrm{GM}}$ is the famous high energy result of Gross and Mende [24]. Recall that this result can be understood from the worldsheet as a saddle point calculation which localized the integration over the string moduli around its extremum value. Of course, the full integral is not just the saddle but is obtained by including all the quantum fluctuations around that classical configuration. That is what comes with the Bernoulli numbers.

There are probably interesting games one could play about understanding the resurgence properties of these infinite sum representations and how the various low energy zeta function coefficients resum into the high energy moduli space expansion Bernouli numbers and vice-versa. After all, as explained in the text, we end up resumming the low energy expansion (C.2) when coming from SUGRA while we will probably land on the high energy expansion (C.4) when arriving from the large classical strings side. As such, understanding the interpolation between these two regimes is key to taming the full interpolation in figure 1 discussed above.

Amusingly this interpolation is very reminiscent of the dressing phase interpolation in the $N=4 \mathrm{SYM}$ spin chain. As explained in $[32,33]$ the weak coupling expansion there is full of Zeta functions while the strong coupling expansion is populated by Bernouli numbers which turn out to be also given by (analytically continued) zeta functions. Mathematically, the reason for this similarity is clear: both Virasoro-Shapiro and the AdS/CFT dressing phase have Gamma functions as their building blocks [34]. Would be very nice to find a physical analogy beyond this technical observation 


\section{C.2 More on the large $p$ limit}

Here we give more details about the flat space limit of the VS amplitude in $A d S_{5} \times S^{5}$, as we mentioned in section 3.2. Recall the usual flat space limit formula (in $d$ dimensions) of Penedones [5],

$$
\mathcal{A}_{\mathrm{VS}}(s, t)=C \lim _{R \rightarrow \infty} \oint \frac{d z}{2 \pi i} z^{-\left(\Sigma-\frac{d}{2}+4\right)} e^{z} \mathcal{M}\left(\frac{R^{2}}{4 z} s, \frac{R^{2}}{4 z} t, \tilde{s}, \tilde{t}\right)
$$

where $C$ is a normalisation. The exponent $\Sigma-\frac{d}{2}+4$ is correct for taking into account the dynamical correlator. We use (C.5) to fix the top degree monomials in $s$ and $t$ in the $A d S_{5}$ part of the tree level amplitude, order by order in $1 / \sqrt{\lambda}$,

$$
\left.\mathcal{M}_{\vec{p}}(s, t, \tilde{s}, \tilde{t})\right|_{\text {genus }=0}=\frac{1}{(\mathbf{s}+1)(\mathbf{t}+1)(\mathbf{u}+1)}+\sum_{h=0}^{\infty}\left(\frac{1}{\sqrt{\lambda}}\right)^{h+3} \mathcal{V}_{\vec{p}}^{(1, h)}(s, t, \tilde{s}, \tilde{t})
$$

where $\mathcal{V}^{(1, h)}$ is a polynomial of degree $h$, against the Virasoro Shapiro amplitude.

$$
\mathcal{A}_{\mathrm{VS}}(s, t)=-\frac{1}{s t(s+t)}\left(1+\sum_{h=0}^{\infty}\left(\frac{\alpha^{\prime}}{4}\right)^{h+3} \mathcal{A}_{\mathrm{VS}}^{(h+3)}(s, t)\right)
$$

where the $\mathcal{A}_{\mathrm{VS}}^{(h)}$ are known.

Computing the integral in (C.5) amounts to solve an inverse $\Gamma$ function integral and results in some Pochhammers, after diving by the normalisation $C=\left(\frac{R}{2}\right)^{6} \Gamma\left[\Sigma-\frac{d}{2}+1\right]$. Then,

$$
\lim _{s, t, \rightarrow \infty}\left(\frac{1}{\sqrt{\lambda}}\right)^{h+3} \mathcal{V}^{(1, h)}(s, t, \tilde{s}, \tilde{t})=\left(\frac{\alpha^{\prime}}{R^{2}}\right)^{h+3} \frac{(\Sigma-1)_{h+3} \mathcal{A}_{\mathrm{VS}}^{(h+3)}(s, t)}{s t(-s-t)} .
$$

Notice that the r.h.s. will always be polynomial. A well known feature of the VirasoroShapiro amplitude.

Switching to the large $p$ limit we restore the dependence on $\tilde{s}$ and $\tilde{t}$ introducing the bold font variables, and because $\mathcal{A}_{\mathrm{VS}}^{(h)}$ is a homogeneous polynomial, we arrive at

$$
\lim _{p \rightarrow \infty} \mathcal{V}^{(1, h)}(s, t, \tilde{s}, \tilde{t})=\left[-\frac{\mathcal{A}_{\mathrm{VS}}^{(h+3)}(\Sigma s, \Sigma t)}{s t(s+t)}\right]_{\substack{s \rightarrow \mathbf{s} \\ t \rightarrow \mathbf{t} \\ u \rightarrow \mathbf{u}}}
$$

where the term in between [...] is supposed to be simplified first, as usual for the flat VS amplitude, and the result, which is now a polynomial of degree $h$, covariantised w.r.t. the bold font variables of $A d S_{5} \times S^{5}$, which are such that $\mathbf{s}+\mathbf{t}+\mathbf{u}=-4$. This is also what we recover, independently, by looking at the $\zeta_{5}$ contributions of the full $A d S_{5} \times S^{5}$ amplitude in (3.12), rewritten under the large $p$ stratification.

At the saddle point $\mathbf{s}_{\mathrm{cl}}+\mathbf{t}_{\mathrm{cl}}+\mathbf{u}_{\mathrm{cl}}=0$, the series in the large $p$ limit can now resummed, in the very same way as for $\mathcal{A}_{\mathrm{VS}}$. We obtain the nice result in terms of $\Gamma$ functions

$$
\left.\lim _{p \rightarrow \infty} \mathcal{M}\left(s_{\mathrm{cl}}, t_{\mathrm{cl}}, \tilde{s}_{\mathrm{cl}}, \tilde{t}_{\mathrm{cl}}\right)\right|_{\text {genus }=0}=\frac{1}{\mathbf{s}_{\mathrm{cl}} \mathbf{t}_{\mathrm{cl}} \mathbf{u}_{\mathrm{cl}}} \frac{\Gamma\left[1-\frac{\Sigma}{\sqrt{\lambda}} \mathbf{s}_{\mathrm{cl}}\right] \Gamma\left[1-\frac{\Sigma}{\sqrt{\lambda}} \mathbf{t}_{\mathrm{cl}}\right] \Gamma\left[1-\frac{\Sigma}{\sqrt{\lambda}} \mathbf{u}_{\mathrm{cl}}\right]}{\Gamma\left[1+\frac{\Sigma}{\sqrt{\lambda}} \mathbf{s}_{\mathrm{cl}}\right] \Gamma\left[1+\frac{\Sigma}{\sqrt{\lambda}} \mathbf{t}_{\mathrm{cl}}\right] \Gamma\left[1+\frac{\Sigma}{\sqrt{\lambda}} \mathbf{u}_{\mathrm{cl}}\right]}
$$

which is quoted in section in 3.2 . 


\section{Leading discontinuities from $\widehat{\mathcal{D}}(\vec{p})$ and $\Delta^{(8)}$}

In section 4 we constructed the generic prepotential $\mathcal{P}_{p_{1} p_{2} p_{3} p_{4}}$ by acting on $\mathcal{P}_{2222}$ with the operators $\widehat{\mathcal{D}}_{\tilde{s}, \tilde{t}}(\vec{p})$. For completeness we repeat $\mathcal{P}_{2222}$ in our conventions. In position space,

$$
\begin{aligned}
\mathcal{P}_{2222}= & \frac{\mathcal{P}_{2222}^{2,2^{-}}}{\left(x_{1}-x_{2}\right)^{7}}\left(\operatorname{Li}_{1}^{2}\left(x_{1}\right)-\mathrm{Li}_{1}^{2}\left(x_{2}\right)\right)+\frac{\mathcal{P}_{2222}^{1,2^{-}}}{\left(x_{1}-x_{2}\right)^{7}}\left(\operatorname{Li}_{2}\left(x_{1}\right)-\mathrm{Li}_{2}\left(x_{2}\right)\right) \\
& \frac{\mathcal{P}_{2222}^{1^{-}}}{\left(x_{1}-x_{2}\right)^{7}}\left(\operatorname{Li}_{1}\left(x_{1}\right)-\operatorname{Li}_{1}\left(x_{2}\right)\right)+\frac{\mathcal{P}_{2222}^{1^{+}}}{\left(x_{1}-x_{2}\right)^{6}} \log (v)+\frac{\mathcal{P}_{2222}^{0}}{\left(x_{1}-x_{2}\right)^{6}}
\end{aligned}
$$

where the various polynomials $\mathcal{P}_{2222}^{\text {basis element,weight }}$ are

$$
\begin{aligned}
& \mathcal{P}_{2222}^{2,2^{-}}=+\frac{1}{4} U^{4} V^{2}(1-U-V) \\
& \mathcal{P}_{2222}^{1,2^{-}}=-\frac{1}{2} U^{4}\left((1-V)^{2}(1+V)+\mathrm{U}\left(1+V^{2}\right)\right) \\
& \mathcal{P}_{2222}^{1^{-}}=-\frac{1}{24} U^{3}\left(U^{4}+(1-V)^{4}-4 U^{3}(1+V)+8 \mathrm{U}(1-V)^{2}(1+V)-2 U^{2}\left(3-8 V+3 V^{2}\right)\right) \\
& \mathcal{P}_{2222}^{1^{+}}=+\frac{1}{24} U^{3}(1-V)\left(U^{2}+(1-V)^{2}+10 \mathrm{U}(1+V)\right) \\
& \mathcal{P}_{2222}^{0}=-\frac{1}{12} U^{4}\left(2 U^{2}-7(1-V)^{2}-7 \mathrm{U}(1+V)\right)
\end{aligned}
$$

This is the prepotential for the amplitude (normalised as in previous section).

Mellinising the above result we find

$$
\mathcal{P}_{2222}(U, V)=-\iint d s d t(-U)^{s+4} V^{t} \frac{\Gamma[-s]}{\Gamma[s+1]} \Gamma[-t]^{2} \Gamma[-u]^{2} \times \mathcal{N}_{2222}(s, t)
$$

where the amplitude $\mathcal{N}_{2222}(s, t)$ is (4.4). The result we gave next, in (4.7), follows from a pencil and paper computation, as we show here below.

The operators $\widehat{\mathcal{D}}_{\tilde{s}, \tilde{t}}(\vec{p})$ in (4.1)-(4.2), when acting on the Mellin integrand of $\mathcal{P}_{2222}$, only act on the monomial $U^{4+s} V^{t}$, returning a combination of Pochhammers in $s$ and $t$, which can be straightforwardly turned into $\Gamma$ functions. Since the denominator of $\widehat{\mathcal{D}}_{\tilde{s}, \tilde{t}}(\vec{p})$ is immediately recognisable as the denominator of $\Gamma_{\otimes}$, we shall focus on the numerator, by defining

$$
\widehat{\mathcal{D}}_{\tilde{s}, \tilde{t}}(\vec{p})=\frac{(-)^{c_{t}+c_{s}} \widetilde{\mathcal{D}}_{\tilde{s}, \tilde{t}}(\vec{p})}{\Gamma[1+\tilde{s}] \Gamma[1+\tilde{t}] \Gamma[1+\tilde{u}] \Gamma\left[1+\tilde{s}+c_{s}\right] \Gamma\left[1+\tilde{t}+c_{t}\right] \Gamma\left[1+\tilde{u}+c_{u}\right]} .
$$

Then, acting on the integrand of $\mathcal{P}_{2222}$ we find.

$$
\begin{aligned}
& U^{-4-s} V^{-t} \widetilde{\mathcal{D}}_{\tilde{s}, \tilde{t}}(\vec{p})\left(U^{4+s} V^{t}\right)= \\
& (s+1-\tilde{s}) \tilde{s}\left(s+1-\tilde{s}-c_{s}\right) \tilde{s}+c_{s}(t+1-\tilde{t})_{\tilde{t}}\left(t+1-\tilde{t}+c_{t}\right)_{\tilde{t}+c_{t}}(s+t+4)_{\tilde{u}}(s+t+4)_{\tilde{u}+c_{u}}= \\
& \frac{\Gamma[s+1]^{2} \Gamma[t+1]^{2} \Gamma[s+t+4+\tilde{u}] \Gamma\left[s+t+4+\tilde{u}+c_{u}\right]}{\Gamma[s+1-\tilde{s}] \Gamma\left[s+1-\tilde{s}-c_{s}\right] \Gamma[t+1-\tilde{t}] \Gamma\left[t+1-\tilde{t}-c_{t}\right] \Gamma[s+t+4]^{2}} .
\end{aligned}
$$


The next step is to put together $\widetilde{\mathcal{D}}(\vec{p})\left(U^{4+s} V^{t}\right)$ with $\Gamma[-s] \Gamma[-t]^{2} \Gamma[s+t+4]^{2} / \Gamma[s+1]$, where the latter is part of the integrand of $\mathcal{P}_{2222}$. Let us split the computation in the three channels:

u-channel. This is the simplest,

$$
\frac{\Gamma[s+t+4+\tilde{u}] \Gamma\left[s+t+4+\tilde{u}+c_{u}\right]}{\Gamma[s+t+4]^{2}} \times \Gamma[s+t+4]^{2}=\Gamma[s+t+4+\tilde{u}] \Gamma\left[s+t+4+\tilde{u}+c_{u}\right] .
$$

Notice that $s+t+4+\tilde{u}=(s-\tilde{s})+(t-\tilde{t})+p_{3}+2$.

t-channel. In this case we will need a reflection identity,

$$
(-)^{c_{t}} V^{t-\tilde{t}} \frac{\Gamma[t+1]^{2}}{\Gamma[t+1-\tilde{t}] \Gamma\left[t+1-\tilde{t}-c_{t}\right]} \times \Gamma[-t]^{2}=V^{t-\tilde{t}} \Gamma[\tilde{t}-t] \Gamma\left[\tilde{t}+c_{t}-t\right]
$$

where on the r.h.s. we used $\Gamma\left[t-n_{i}\right]=(-)^{n_{i}-1} \Gamma[-t] \Gamma[t+1] / \Gamma\left[n_{i}+1-t\right]$ with $n_{1}=\tilde{t}-1$ and $n_{2}=\tilde{t}+c_{t}-1$ (assumed to be integers, as it should).

s-channel. Similarly to the previous case,

$$
(-)^{c_{s}} U^{2+s-\tilde{s}} \frac{\Gamma[s+1]^{2}}{\Gamma[s+1-\tilde{s}] \Gamma\left[s+1-\tilde{s}-c_{s}\right]} \times \frac{\Gamma[-s]}{\Gamma[s+1]}=U^{2+s-\tilde{s}} \frac{\Gamma[\tilde{s}-s] \Gamma\left[\tilde{s}+c_{s}-s\right]}{\Gamma[-s] \Gamma[s+1]} .
$$

The gamma function kernel on the r.h.s. should only have simple poles, whose residue would be such that it reproduces that of $\Gamma_{\otimes}$. The idea is that $\tilde{s}$ and $\tilde{s}+c_{s}$ are both integers, therefore we can turn around the reflection identity, in two way

$$
\begin{aligned}
\Gamma[-s] \Gamma[s+1] & =(-)^{\tilde{s}+c_{s}-1} \Gamma\left[\tilde{s}+c_{s}-s\right] \Gamma\left[s-c_{s}-\tilde{s}+1\right] \\
& =(-)^{\tilde{s}-1} \Gamma[\tilde{s}-s] \Gamma[s-\tilde{s}+1]
\end{aligned}
$$

depending on the situation. What fixes this is the leading twist of the correlator $U^{\max \left(0, c_{s}\right)}$. Therefore, if $c_{s}<0$ we consider the first line, otherwise the second line. To consider both cases at once it is useful to introduce $\max \left(0, c_{s}\right)$.

Let us illustrate the final result in the case $c_{s}<0$, just for concreteness,

$$
\begin{aligned}
\mathcal{P}_{p_{1} p_{2} p_{3} p_{4}}= & (U \tilde{U})^{2} \sum_{T} \int U^{s-\tilde{s}} V^{t-\tilde{t}} \tilde{U}^{\tilde{s}} \tilde{V}^{\tilde{t}} \times \mathcal{N}_{2222}(s, t) \times(-)^{s+1} \frac{\Gamma[\tilde{s}-s]}{(-)^{\tilde{s}+c_{s}-1} \Gamma\left[s-\tilde{s}-c_{s}+1\right]} \times \\
& \frac{\Gamma[\tilde{t}-t] \Gamma\left[\tilde{t}-t+c_{t}\right] \Gamma\left[s-\tilde{s}+t-\tilde{t}+p_{3}+2\right] \Gamma\left[s-\tilde{s}+t-\tilde{t}+p_{3}+2+c_{u}\right]}{\Gamma[1+\tilde{s}] \Gamma[1+\tilde{t}] \Gamma[1+\tilde{u}] \Gamma\left[1+\tilde{s}+c_{s}\right] \Gamma\left[1+\tilde{t}+c_{t}\right] \Gamma\left[1+\tilde{u}+c_{u}\right]} .
\end{aligned}
$$

Finally, by changing variables $s \rightarrow s+\tilde{s}$ and $t \rightarrow t+\tilde{t}$ and defining $u=-s-t-p_{3}-2$, we get

$$
\begin{aligned}
\mathcal{P}_{p_{1} p_{2} p_{3} p_{4}}=(-)^{c_{s}}(U \tilde{U})^{2} & \sum_{T} \int(-U)^{s} V^{t} \tilde{U}^{\tilde{s}} \tilde{V}^{\tilde{t}} \times \mathcal{N}_{2222}(\mathbf{s}, \mathbf{t}) \frac{\Gamma[-s]}{\Gamma\left[s-c_{s}+1\right]} \times \\
& \frac{\Gamma[-t] \Gamma\left[-t+c_{t}\right] \Gamma[-u] \Gamma\left[-u+c_{u}\right]}{\Gamma[1+\tilde{s}] \Gamma[1+\tilde{t}] \Gamma[1+\tilde{u}] \Gamma\left[1+\tilde{s}+c_{s}\right] \Gamma\left[1+\tilde{t}+c_{t}\right] \Gamma\left[1+\tilde{u}+c_{u}\right]} .
\end{aligned}
$$


Notice, $\mathcal{N}_{2222}$ is evaluated in $\mathbf{s}=s+\tilde{s}$ and $\mathbf{t}=t+\tilde{s}$ and $\Gamma_{\otimes}$ is essentially manifest, we still have simple poles in $s$ but the residue is the one $\Gamma_{\otimes}$ would induce.

Let us move now to $\Delta^{(8)}$. The eight-order differential operator $\Delta^{(8)}$ is best written in the form $[20]$

$$
\begin{aligned}
\delta_{\left[p_{21}, p_{43}\right]}^{(8)} & =\frac{x_{1} x_{2} y_{1} y_{2}}{\left(x_{1}-x_{2}\right)\left(y_{1}-y_{2}\right)} \prod_{i, j=1}^{2}\left(\mathbf{C}_{x_{i}}^{\left[+\frac{p_{21}}{2},+\frac{p_{43}}{2}, 0\right]}-\mathbf{C}_{y_{j}}^{\left[-\frac{p_{21}}{2},-\frac{p_{43}}{2}, 0\right]}\right) \frac{\left(x_{1}-x_{2}\right)\left(y_{1}-y_{2}\right)}{x_{1} x_{2} y_{1} y_{2}} \\
\Delta_{\left[p_{21}, p_{43}\right]}^{(8)} & =\left(\frac{U}{\tilde{U}}\right)^{-\frac{p_{4}+p_{3}}{2}} \delta_{\left[p_{21}, p_{43}\right]}^{(8)}\left(\frac{U}{\tilde{U}}\right)^{\frac{p_{4}+p_{3}}{2}}
\end{aligned}
$$

where $\mathbf{C}_{z}^{[\alpha, \beta, \gamma]}$ is the elementary $2 d$ casimir $\mathbf{C}_{z}^{[\alpha, \beta, \gamma]}=z^{2}(1-z) \partial_{z}^{2}+z(\gamma-(1+\alpha+\beta) z) \partial_{z}-\alpha \beta z$. $\Delta^{(8)}$ depends non trivially on the external charges. It is invariant under the 'flip' symmetry $x_{i} \rightarrow x_{i} /\left(x_{i}-1\right)$ and $y_{i} \rightarrow y_{i} /\left(y_{i}-1\right)$ which is a symmetry of the correlator when the external charges satisfy $p_{21}=0 \| p_{43}=0$.

Continuing with the amplitude we find

$$
\mathcal{A}_{p_{1} p_{2} p_{3} p_{4}}=U^{-2} \tilde{U}^{-2} \Delta^{(8)} \mathcal{P}_{p_{1} p_{2} p_{3} p_{4}} .
$$

The action of $\Delta^{(8)}$ is localised on $U^{2+s} V^{t} \tilde{U}^{\tilde{s}+2} \tilde{V}^{\tilde{t}}$, which appear in the Mellin representation of $\mathcal{P}_{p_{1} p_{2} p_{3} p_{4}}$. It returns

$$
\begin{aligned}
& U^{-2} \tilde{U}^{-2} \Delta^{(8)}\left[U^{2+s} V^{t} \tilde{U}^{\tilde{s}+2} \tilde{V}^{\tilde{t}}\right]= \\
& U^{s} V^{t} \tilde{U}^{\tilde{s}} \tilde{V}^{\tilde{t}}\left[\sum_{0 \leq i+j \leq 4}\left(\frac{1}{2} p_{21}\right)^{i}\left(\frac{1}{2} p_{34}\right)^{j} \Omega_{i j}\left(U, V, \tilde{U}, \tilde{V}, s, t, \tilde{s}, \tilde{t}, p_{3}\right)\right]
\end{aligned}
$$

where in the second line we also used $\frac{p_{4}+p_{3}}{2}=-\frac{1}{2} p_{34}+p_{3}$. The $\Omega_{i j}$ can be expanded as

$$
\Omega_{i j}\left(U, V, \tilde{U}, \tilde{V}, s, t, \tilde{s}, \tilde{t}, p_{3}\right)=\sum_{\substack{0 \leq m+n \leq 4 \\ 0 \leq \tilde{m}+\tilde{n} \leq 4}} A_{i j}[m, n, \tilde{m}, \tilde{n}]\left(s, t, \tilde{s}, \tilde{t}, p_{3}\right) U^{m} V^{n-2} \tilde{U}^{\tilde{n}} \tilde{V}^{\tilde{m}-2}
$$

and the $A_{i j}[m, n, \tilde{m}, \tilde{n}]\left(s, t, \tilde{s}, \tilde{t}, p_{3}\right)$ are attached in the supplementary material. Notice the overall $V^{-2} \tilde{V}^{-2}$. For each $i, j$, i.e. powers of $p_{21}$ and $p_{34}$, there are 225 terms. This means that when we Mellinise a given $\Omega_{i j}$ we find a shift-operator with 225 terms. These shifts act on the Mellin variables $\mathbf{s}$ and $\mathbf{t}$, i.e. those in $\mathcal{N}_{2222}(\mathbf{s}, \mathbf{t})$, as we showed above. However, there are only 25 different shifts and these are the ones we collect. Out of an $\Omega_{i j}\left(U, \ldots, p_{3}\right)$ we obtain a shift operator

$$
\widehat{\Omega}_{i j}(a, b) ; \quad-4 \leq a \leq 0 ; \quad-3-a \leq b \leq 3
$$

where $a$ and $b$ label the shifts.

There is something interesting to point out about the $\widehat{\Omega}_{i j}(a, b)$. The coefficients of these shift-operators are rational functions, since they come directly from rearranging 
" $\Gamma_{\otimes}$ ", which has numerator and denominator. However, when we sum over $i, j$ we find polynomials. In section 4.2 we used,

$$
\delta_{p_{1} p_{2} p_{3} p_{4}}(a, b)=\sum_{i, j} \widehat{\Omega}_{i j}(a, b)
$$

which are indeed polynomials in $s, t, \tilde{s}, \tilde{t}, p_{i=1,2,3,4}$, and are attached in the supplementary material for illustration. ${ }^{22}$

The relation between $\Omega^{(8)}\left(U, \ldots p_{3}\right)$ and $\Omega^{(8)}\left(U, \ldots, p_{3}\right)$ evaluated at $\{U, \tilde{U}, V, \tilde{V}\} \rightarrow$ $\{U / V, \tilde{U} / \tilde{V}, 1 / V, 1 / \tilde{V}\}$, can be seen as follows,

$$
A_{0 j}\left(m, n, \tilde{m}, \tilde{n}, s, t, \tilde{s}, \tilde{t}, p_{3}\right)=+A_{0 j}\left(m,-n-m+4, \tilde{m},-\tilde{n}-\tilde{m}+4, s, u, \tilde{s}, \tilde{u}, p_{3}\right)
$$

and also

$$
A_{i j \neq 0}\left(m, n, \tilde{m}, \tilde{n}, s, t, \tilde{s}, \tilde{t}, p_{3}\right)=-A_{i j \neq 0}\left(m,-n-m+4, \tilde{m},-\tilde{n}-\tilde{m}+4, s, u, \tilde{s}, \tilde{u}, p_{3}\right) .
$$

\section{D.1 Leading log at two-loop}

The scaling with $p$ of the Mellin amplitude, generalised to the $\ell$-loop, gives the following scheme:

$$
\begin{aligned}
\lim _{p \rightarrow \infty} \mathcal{M}_{\vec{p}}^{\ell-\text { loop }}\left(s_{\mathrm{cl}}, t_{\mathrm{cl}}, \tilde{s}_{\mathrm{cl}}, \tilde{t}_{\mathrm{cl}}\right)=\frac{1}{\mathbf{s}_{\mathrm{cl}} \mathbf{t}_{\mathrm{cl}} \mathbf{u}_{\mathrm{cl}}} \times\left(\frac{\Sigma^{4} \mathbf{s}_{\mathrm{cl}}^{4}}{N^{2}}\right)^{\ell} \\
{\left[\sum_{0 \leq d \leq 2 \ell} \text { lim-Transcendetal } \mathbf{l}_{\ell, d}\left(\mathbf{s}_{\mathrm{cl}}, \mathbf{t}_{\mathrm{cl}}\right) \times \operatorname{Rational}_{\ell, d}\left(\frac{\mathbf{t}_{\mathrm{cl}}}{\mathbf{s}_{\mathrm{cl}}}\right)+\text { crossing }\right] }
\end{aligned}
$$

as we explained around (4.41).

We can test our scheme at two-loops, for the top-weigh function Rational 2,6 , since this is given only by the triple discontinuity, which we know [15, 20]. In fact, we can also illustrate with a short computation how to organise the top-weight construction of the full two-loop amplitude. At two-loops in position space, the top-weight term is accompanied by a weight six function, and there are six independent such pure functions [57], i.e. no symmetry. None of them has the $\log ^{3} u \log ^{3} v$ contribution, rather we find at most a $\log ^{3} u \log ^{2} v f_{1}(u, v)$ in one orientation. We pick this one, and even though we don't know all the details of the full amplitude, we can just focus on the Mellin transform of its coefficient function, which is a rational function and will give us Rational 2,6 in the end. We Mellinise that coefficient function for the stress-energy tensor correlator and we obtain $\mathcal{T}^{(6)}(s, t)$. Then, we adapt the same procedure that led us from $\mathcal{P}_{2222}$ to $\mathcal{P}_{p_{1} p_{2} p_{3} p_{4}}$ at oneloop. Thus we obtain a Mellin amplitude which includes $\Gamma_{\otimes}$ with two sin flips, because we are studying a rational function in position space, and $\mathcal{T}^{(6)}(\mathbf{s}, \mathbf{t})$. In sum,

$$
\begin{aligned}
\mathcal{T}^{(6)}(s, t) & =-\frac{1}{21600} \frac{120+99 s+21 s^{2}+29 t+12 s t+t^{2}}{(s+1)(s+2)(t+1)(t+2)(t+3)} \\
\text { Rational }_{2,6} & =\mathbf{s}_{\mathrm{cl}} \mathbf{t}_{\mathrm{cl}} \mathbf{u}_{\mathrm{cl}} \lim _{p \rightarrow \infty} \mathcal{T}^{(6)}\left(\mathbf{s}_{\mathrm{cl}}, \mathbf{t}_{\mathrm{cl}}\right)=-\frac{21\left(\mathbf{s}_{\mathrm{cl}}\right)^{2} \mathbf{u}_{\mathrm{cl}}+\left(\mathbf{t}_{\mathrm{cl}}\right)^{2} \mathbf{u}_{\mathrm{cl}}+12 \mathbf{s}_{\mathrm{cl}} \mathbf{t}_{\mathrm{cl}} \mathbf{u}_{\mathrm{cl}}}{21600\left(\mathbf{s}_{\mathrm{cl}}\right)\left(\mathbf{t}_{\mathrm{cl}}\right)^{2}} .
\end{aligned}
$$

\footnotetext{
${ }^{22}$ In the supplementary material we use $S$ and $T$ instead of $\tilde{s}$ and $\tilde{t}$, just for convenience.
} 
Indeed we find that Rational ${ }_{2,6}$ has degree zero under the large $p$ limit, and we expect the action of $\Delta^{(8)} \Delta^{(8)}$ to bring $\Sigma^{8} \mathbf{s}_{\mathrm{cl}}^{8}$. The matching with the ten-dimensional double boxes at top-weight starts from (D.24) and the Mellin transform of the weight six function [57], which we postpone to a future work. Our strategy complements the infinite sum discussion of [43].

Open Access. This article is distributed under the terms of the Creative Commons Attribution License (CC-BY 4.0), which permits any use, distribution and reproduction in any medium, provided the original author(s) and source are credited.

\section{References}

[1] L. Susskind, Holography in the flat space limit, AIP Conf. Proc. 493 (1999) 98 [hep-th/9901079] [INSPIRE].

[2] J. Polchinski, S matrices from AdS space-time, hep-th/9901076 [INSPIRE].

[3] S.B. Giddings, Flat space scattering and bulk locality in the AdS/CFT correspondence, Phys. Rev. D 61 (2000) 106008 [hep-th/9907129] [INSPIRE].

[4] I. Heemskerk, J. Penedones, J. Polchinski and J. Sully, Holography from Conformal Field Theory, JHEP 10 (2009) 079 [arXiv:0907.0151] [INSPIRE].

[5] J. Penedones, Writing CFT correlation functions as AdS scattering amplitudes, JHEP 03 (2011) 025 [arXiv: 1011.1485] [INSPIRE].

[6] A.L. Fitzpatrick and J. Kaplan, Scattering States in AdS/CFT, arXiv:1104.2597 [INSPIRE].

[7] A.L. Fitzpatrick and J. Kaplan, Analyticity and the Holographic S-matrix, JHEP 10 (2012) 127 [arXiv:1111.6972] [INSPIRE].

[8] V. Gonçalves, Four point function of $\mathcal{N}=4$ stress-tensor multiplet at strong coupling, JHEP 04 (2015) 150 [arXiv:1411.1675] [INSPIRE].

[9] S. Komatsu, M.F. Paulos, B.C. Van Rees and X. Zhao, Landau diagrams in AdS and S-matrices from conformal correlators, JHEP 11 (2020) 046 [arXiv:2007.13745] [INSPIRE].

[10] L. Rastelli and X. Zhou, Mellin amplitudes for $A d S_{5} \times S^{5}$, Phys. Rev. Lett. 118 (2017) 091602 [arXiv: 1608.06624] [INSPIRE].

[11] L.F. Alday and A. Bissi, Loop Corrections to Supergravity on $A d S_{5} \times S^{5}$, Phys. Rev. Lett. 119 (2017) 171601 [arXiv:1706.02388] [INSPIRE].

[12] F. Aprile, J.M. Drummond, P. Heslop and H. Paul, Quantum Gravity from Conformal Field Theory, JHEP 01 (2018) 035 [arXiv: 1706.02822] [INSPIRE].

[13] F. Aprile, J.M. Drummond, P. Heslop and H. Paul, Unmixing Supergravity, JHEP 02 (2018) 133 [arXiv: 1706.08456] [INSPIRE].

[14] F. Aprile, J.M. Drummond, P. Heslop and H. Paul, Loop corrections for Kaluza-Klein AdS amplitudes, JHEP 05 (2018) 056 [arXiv: 1711.03903] [INSPIRE].

[15] F. Aprile, J.M. Drummond, P. Heslop and H. Paul, Double-trace spectrum of $N=4$ supersymmetric Yang-Mills theory at strong coupling, Phys. Rev. D 98 (2018) 126008 [arXiv: 1802.06889] [INSPIRE]. 
[16] F. Aprile, J.M. Drummond, P. Heslop and H. Paul, One-loop amplitudes in $A d S_{5} \times S^{5}$ supergravity from $\mathcal{N}=4$ SYM at strong coupling, JHEP 03 (2020) 190 [arXiv:1912.01047] [INSPIRE].

[17] F. Aprile et al., Single particle operators and their correlators in free $\mathcal{N}=4$ SYM, JHEP 11 (2020) 072 [arXiv : 2007.09395] [INSPIRE].

[18] L. Rastelli and X. Zhou, How to Succeed at Holographic Correlators Without Really Trying, JHEP 04 (2018) 014 [arXiv:1710.05923] [InSPIRE].

[19] L.F. Alday and S. Caron-Huot, Gravitational S-matrix from CFT dispersion relations, JHEP 12 (2018) 017 [arXiv: 1711.02031] [INSPIRE].

[20] S. Caron-Huot and A.-K. Trinh, All tree-level correlators in AdS $S_{5} \times S_{5}$ supergravity: hidden ten-dimensional conformal symmetry, JHEP 01 (2019) 196 [arXiv: 1809.09173] [INSPIRE].

[21] F.A. Dolan and H. Osborn, Conformal partial wave expansions for $N=4$ chiral four point functions, Annals Phys. 321 (2006) 581 [hep-th/0412335] [INSPIRE].

[22] F.A. Dolan, M. Nirschl and H. Osborn, Conjectures for large $N$ superconformal $N=4$ chiral primary four point functions, Nucl. Phys. B 749 (2006) 109 [hep-th/0601148] [INSPIRE].

[23] J.A. Minahan, Holographic three-point functions for short operators, JHEP 07 (2012) 187 [arXiv:1206.3129] [INSPIRE].

[24] D.J. Gross and P.F. Mende, The High-Energy Behavior of String Scattering Amplitudes, Phys. Lett. B 197 (1987) 129 [InSPIRE].

[25] B. Basso, F. Coronado, S. Komatsu, H.T. Lam, P. Vieira and D.-l. Zhong, Asymptotic Four Point Functions, JHEP 07 (2019) 082 [arXiv:1701.04462] [INSPIRE].

[26] F. Coronado, Perturbative four-point functions in planar $\mathcal{N}=4 S Y M$ from hexagonalization, JHEP 01 (2019) 056 [arXiv:1811.00467] [INSPIRE].

[27] F. Coronado, Bootstrapping the Simplest Correlator in Planar $\mathcal{N}=4$ Supersymmetric Yang-Mills Theory to All Loops, Phys. Rev. Lett. 124 (2020) 171601 [arXiv:1811.03282] [INSPIRE].

[28] T. Fleury and S. Komatsu, Hexagonalization of Correlation Functions, JHEP 01 (2017) 130 [arXiv: 1611.05577] [INSPIRE].

[29] B. Basso, S. Komatsu and P. Vieira, Structure Constants and Integrable Bootstrap in Planar $N=4 S Y M$ Theory, arXiv:1505.06745 [INSPIRE].

[30] I. Kostov, V.B. Petkova and D. Serban, Determinant Formula for the Octagon Form Factor in $N=4$ Supersymmetric Yang-Mills Theory, Phys. Rev. Lett. 122 (2019) 231601 [arXiv: 1903.05038] [INSPIRE].

[31] T. Bargheer, F. Coronado and P. Vieira, Octagons I: Combinatorics and Non-Planar Resummations, JHEP 08 (2019) 162 [arXiv: 1904.00965] [INSPIRE].

[32] N. Beisert, B. Eden and M. Staudacher, Transcendentality and Crossing, J. Stat. Mech. 0701 (2007) P01021 [hep-th/0610251] [INSPIRE].

[33] N. Beisert, R. Hernandez and E. Lopez, A Crossing-symmetric phase for $A d S_{5} \times S^{5}$ strings, JHEP 11 (2006) 070 [hep-th/0609044] [INSPIRE].

[34] N. Dorey, D.M. Hofman and J.M. Maldacena, On the Singularities of the Magnon S-matrix, Phys. Rev. D 76 (2007) 025011 [hep-th/0703104] [INSPIRE]. 
[35] T. Bargheer, F. Coronado and P. Vieira, Octagons II: Strong Coupling, arXiv:1909.04077 [INSPIRE].

[36] A.V. Belitsky and G.P. Korchemsky, Exact null octagon, JHEP 05 (2020) 070 [arXiv: 1907.13131] [INSPIRE].

[37] A.V. Belitsky and G.P. Korchemsky, Octagon at finite coupling, JHEP 07 (2020) 219 [arXiv: 2003.01121] [INSPIRE].

[38] A.V. Belitsky and G.P. Korchemsky, Crossing bridges with strong Szego limit theorem, arXiv: 2006.01831 [INSPIRE].

[39] B. Eden, A.C. Petkou, C. Schubert and E. Sokatchev, Partial nonrenormalization of the stress tensor four point function in N=4SYM and AdS/CFT, Nucl. Phys. B 607 (2001) 191 [hep-th/0009106] [INSPIRE].

[40] L.F. Alday, A. Bissi and E. Perlmutter, Genus-One String Amplitudes from Conformal Field Theory, JHEP 06 (2019) 010 [arXiv:1809.10670] [INSPIRE].

[41] L.F. Alday, On Genus-one String Amplitudes on $A d S_{5} \times S^{5}$, arXiv:1812.11783 [INSPIRE].

[42] L.F. Alday and X. Zhou, Simplicity of AdS Supergravity at One Loop, JHEP 09 (2020) 008 [arXiv: 1912.02663] [INSPIRE].

[43] A. Bissi, G. Fardelli and A. Georgoudis, Towards All Loop Supergravity Amplitudes on $A d S_{5} \times S^{5}$, arXiv:2002.04604 [INSPIRE].

[44] D.J. Binder, S.M. Chester, S.S. Pufu and Y. Wang, $\mathcal{N}=4$ Super-Yang-Mills correlators at strong coupling from string theory and localization, JHEP 12 (2019) 119 [arXiv:1902.06263] [INSPIRE].

[45] S.M. Chester, Genus-2 holographic correlator on $A d S_{5} \times S^{5}$ from localization, JHEP 04 (2020) 193 [arXiv : 1908.05247] [inSPIRE].

[46] S.M. Chester, M.B. Green, S.S. Pufu, Y. Wang and C. Wen, Modular invariance in superstring theory from $\mathcal{N}=4$ super-Yang-Mills, JHEP 11 (2020) 016 [arXiv:1912.13365] [INSPIRE].

[47] S.M. Chester and S.S. Pufu, Far Beyond the Planar Limit in Strongly-Coupled $\mathcal{N}=4 S Y M$, arXiv:2003.08412 [INSPIRE].

[48] J.M. Drummond, D. Nandan, H. Paul and K.S. Rigatos, String corrections to AdS amplitudes and the double-trace spectrum of $\mathcal{N}=4$ SYM, JHEP 12 (2019) 173 [arXiv: 1907.00992] [INSPIRE].

[49] J.M. Drummond, H. Paul and M. Santagata, Bootstrapping string theory on $A d S_{5} \times S^{5}$, arXiv: 2004.07282 [INSPIRE].

[50] J.M. Drummond and H. Paul, One-loop string corrections to AdS amplitudes from CFT, arXiv: 1912.07632 [INSPIRE].

[51] N.I. Usyukina and A.I. Davydychev, An Approach to the evaluation of three and four point ladder diagrams, Phys. Lett. B 298 (1993) 363 [InSPIRE].

[52] A.P. Isaev, Multiloop Feynman integrals and conformal quantum mechanics, Nucl. Phys. B 662 (2003) 461 [hep-th/0303056] [INSPIRE].

[53] P. Allendes, B. Kniehl, I. Kondrashuk, E.A.N. Cuello and M.R. Medar, Solution to Bethe-Salpeter equation via Mellin-Barnes transform, Nucl. Phys. B $\mathbf{8 7 0}$ (2013) 243 [arXiv: 1205.6257] [INSPIRE]. 
[54] M.B. Green, J.H. Schwarz and L. Brink, $N=4$ Yang-Mills and $N=8$ Supergravity as Limits of String Theories, Nucl. Phys. B 198 (1982) 474 [InSPIRE].

[55] Z. Bern, L.J. Dixon, D.C. Dunbar, M. Perelstein and J.S. Rozowsky, On the relationship between Yang-Mills theory and gravity and its implication for ultraviolet divergences, Nucl. Phys. B 530 (1998) 401 [hep-th/9802162] [INSPIRE].

[56] M.B. Green, J.G. Russo and P. Vanhove, Low energy expansion of the four-particle genus-one amplitude in type-II superstring theory, JHEP 02 (2008) 020 [arXiv:0801.0322] [INSPIRE].

[57] J.M. Drummond, Generalised ladders and single-valued polylogarithms, JHEP 02 (2013) 092 [arXiv:1207.3824] [INSPIRE].

[58] L.F. Alday and J. Maldacena, Null polygonal Wilson loops and minimal surfaces in Anti-de-Sitter space, JHEP 11 (2009) 082 [arXiv:0904.0663] [INSPIRE].

[59] L.F. Alday, D. Gaiotto and J. Maldacena, Thermodynamic Bubble Ansatz, JHEP 09 (2011) 032 [arXiv: 0911.4708] [INSPIRE].

[60] L.F. Alday, J. Maldacena, A. Sever and P. Vieira, Y-system for Scattering Amplitudes, J. Phys. A 43 (2010) 485401 [arXiv: 1002.2459] [InSPIRE].

[61] R.A. Janik and A. Wereszczynski, Correlation functions of three heavy operators: The AdS contribution, JHEP 12 (2011) 095 [arXiv: 1109.6262] [INSPIRE].

[62] Y. Kazama and S. Komatsu, On holographic three point functions for GKP strings from integrability, JHEP 01 (2012) 110 [Erratum JHEP 06 (2012) 150] [arXiv:1110.3949] [INSPIRE].

[63] Y. Kazama and S. Komatsu, Wave functions and correlation functions for GKP strings from integrability, JHEP 09 (2012) 022 [arXiv:1205.6060] [INSPIRE].

[64] Y. Kazama and S. Komatsu, Three-point functions in the $\mathrm{SU}(2)$ sector at strong coupling, JHEP 03 (2014) 052 [arXiv: 1312.3727] [INSPIRE].

[65] S. Komatsu, Liouville theory, AdS $S_{2}$ string, and three-point functions, J. Phys. A 53 (2020) 283002 [arXiv: 1908.03219] [INSPIRE].

[66] J. Caetano and J. Toledo, $\chi$-systems for correlation functions, JHEP 01 (2019) 050 [arXiv: 1208.4548] [INSPIRE].

[67] R. Doobary and P. Heslop, Superconformal partial waves in Grassmannian field theories, JHEP 12 (2015) 159 [arXiv:1508.03611] [INSPIRE].

[68] R.B. Paris and D. Kamiski, Asymptotics and Mellin-Barnes Integrals, in Encyclopedia of Mathematics and its Applications 85, Cambridge University Press, Cambridge U.K. (2001) and online at https://www.cambridge.org/9780521790017.

[69] C.R. Mafra and O. Schlotterer, All Order $\alpha^{\prime}$ Expansion of One-Loop Open-String Integrals, Phys. Rev. Lett. 124 (2020) 101603 [arXiv: 1908.09848] [INSPIRE].

[70] J.E. Gerken, A. Kleinschmidt and O. Schlotterer, All-order differential equations for one-loop closed-string integrals and modular graph forms, JHEP 01 (2020) 064 [arXiv:1911.03476] [INSPIRE].

[71] L. Rastelli, K. Roumpedakis and X. Zhou, $A d S_{3} \times S^{3}$ Tree-Level Correlators: Hidden Six-Dimensional Conformal Symmetry, JHEP 10 (2019) 140 [arXiv:1905.11983] [INSPIRE].

[72] S. Giusto, R. Russo, A. Tyukov and C. Wen, Holographic correlators in AdS 3 without Witten diagrams, JHEP 09 (2019) 030 [arXiv:1905.12314] [INSPIRE]. 
[73] S. Giusto, R. Russo, A. Tyukov and C. Wen, The CFT 6 origin of all tree-level 4-point correlators in $A d S_{3} \times S^{3}$, Eur. Phys. J. C 80 (2020) 736 [arXiv: 2005.08560] [INSPIRE].

[74] L.F. Alday and X. Zhou, All Tree-Level Correlators for M-theory on $A d S_{7} \times S^{4}$, Phys. Rev. Lett. 125 (2020) 131604 [arXiv:2006.06653] [INSPIRE].

[75] L.F. Alday and X. Zhou, All Holographic Four-Point Functions in All Maximally Supersymmetric CFTs, arXiv:2006.12505 [INSPIRE]. 